\title{
Diagrammatic mass factorization
}

\author{
B. Humpert* \\ Swiss Federal Institute of Technology, Laboratory of High Energy Physics, CH-5234 Villigen, Switzerland \\ W. L. van Neerven \\ National Institute for Nuclear and High Energy Physics, NIKHEF-H, P. O. Box 41882, Amsterdam, The Netherlands
}

(Received 29 June 1981)

\begin{abstract}
We develop an analogy between mass factorization (concerning the mass $\equiv$ collinear singularities) and multiplicative Bogoliubov-Parasiuk-Hepp-Zimmermann renormalization (concerning the ultraviolet singularities) with the role of the renormalization constant taken over by the operator matrix elements. By introducing Zimmermann's forest formula we demonstrate its use in constructing the deep-inelastic (DI)/Drell-Yan (DY) factorized parton cross sections and arrive at an all-order proof of mass factorization. The DY correction term is determined by similar reasonings. The noncancellation of the higherorder QCD infrared singularities is left apart.
\end{abstract}

\section{INTRODUCTION}

Mass factorization ${ }^{1}$ in the framework of perturbative QCD has been proven by several different methods, ${ }^{2}$ most of them following a reasoning similar to the Dyson-Salam proof of (multiplicative) renormalization. ${ }^{3}$ Bethe-Salpeter - type equations are used to remove order by order the overlapping divergences, and the remaining infinities are factorized in the overall wave-function renormalization constant. In this line of thought the authors of Refs. 4 and 5 recently drew the attention to the analogy between renormalization and mass factorization.

In this paper we develop Bogoliubov-ParasiukHepp-Zimmermann (BPHZ) mass factorizationthe analog to BPHZ renormalization. ${ }^{6}$ A sketch of the essential ideas has earlier been given in Ref. 5; we shall clarify and expand on the details. BPHZ renormalization disposes of the ultraviolet (UV) divergences of each Feynman graph by introducing counterterms in the Lagrangian, a procedure which is equivalent to the absorption of all infinities in the renormalization constant. While carrying out mass factorization, the role of the UV singularities is taken over by the mass singularities ( $\equiv$ collinear singularities only) of the deep-inelastic (DI)/DrellYan(DY) operator matrix element. Here we are primarily concerned with the fundamental understanding of BPHZ mass factorization which, by this method, is proven to all orders.

Following the work of Zimmermann, ${ }^{6}$ we introduce the mass-factorization parts and define the reduced parton cross section via Bogoliubov's $R$ operation. All ('t Hooft-Veltman ${ }^{7}$ ) overlapping divergences are in this way removed and its overall counterterm is formed by a Taylor expansion around $-q^{2}=\mu^{2}$. The DI/DY Wilson coefficient then easily follows. This whole procedure is in compact form expressed by the DI and DY forest formulas. The unmodified extension of this technique to the singlet graphs requires the introduction of a (spurious) scalar external source which interacts with the gluons. This step is justified by the fact that all mass singularities are universal, and that the mass-factorized cross sections are specified up to a finite (operator) renormalization only. The $R$ operation is therefore slightly modified and the forest formulas too. The analogous reasoning allows specification of the DY correction term. In our presentation, we gradually increase the level of complexity. Starting from an analogy between BPHZ renormalization and mass factorization, we develop the technique first for nonsinglet graphs and subsequently generalize to singlets. Subsequently, we focus our interest on the IR cancellation in, and the gauge dependence of, gauge theories. Our general arguments are illustrated by explicit examples. Having gained sufficient experience, our study leads to a general proof of BPHZ mass factorization which is valid, graph by graph, to all orders.

Our work is primarily concerned with the mass ( $\equiv$ collinear) singularities and does not contribute to nor clarify the noncancellation of the higherorder QCD infrared (IR) singularities (see our later remarks at the onset of Sec. V). As a result, QCD mass factorization breaks down manifesting itself 
by phenomenological effects whose influence in the higher-twist contributions was recently analyzed in Ref. 8. Our subsequent arguments, however, always apply in those processes in QCD where the Bloch-Nordsieck IR cancellation takes place such as, for instance, in the deep-inelastic scattering processes with only one colored particle in the initial state, or in the Drell-Yan processes with only one or no gluon in the final state.

This paper is organized as follows: In Sec. II, we develop the analogy between mass factorization and multiplicative renormalization. In Sec. III, we classify all mass singularities and introduce the general formalism for BPHZ mass factorization of the nonsinglet DI/DY cut graphs. In particular, we explain the $R$ operation, the Taylor expansion, and subsequently Zimmermann's forest formula. Several examples illustrate the application of this technique. In Sec. IV, BPHZ mass factorization is extended to the singlet graphs. A spurious scalar external source is introduced which interacts with the gluons, and the $R$ operation and forest formulas are slightly modified. Section $V$ is reserved for the problems typical in gauge theories such as the IR singularities and the gauge choice. The DY correction term can be evaluated by completely analogous arguments; Sec. VI presents our arguments. Using BPHZ techniques, we prove in Sec. VII mass factorization to all orders. Section VIII is reserved for our summary and conclusions.

\section{ANALOGY: MULTIPLICATIVE RENORMALIZATION-MASS FACTORIZATION}

In this section, we develop the analogy between multiplicative renormalization and mass factorization. Limiting ourselves to the essential reasoning, we omit all additional complications, characteristic for gauge theories, such as spin, mixing, and color. $\left(\Phi^{3}\right)_{6}$ theory therefore seems to us a good toy model to illustrate our arguments.

Suppose a one-particle-irreducible (1PI) truncated Green's function is calculated in a renormalizable theory. Take, for example, a two-point Green's function. Its unrenormalized form, denoted by $\Gamma(p, M, g(M))$, depends on the regulator mass $M$, the external momentum $p$, and the bare coupling constant $g(M)$. A subtraction at $p^{2}=\mu^{2}$ leads to the renormalized Green's function $\Gamma_{R}(p, \mu, g(\mu))$. The renormalized and the unrenormalized Green's functions are then related by multiplicative renormalization as

$$
\begin{aligned}
\Gamma_{R}(p, \mu, g(\mu))= & Z^{-1}(M, \mu, g(\mu)) \\
& \times \Gamma(p, M, g(M)) .
\end{aligned}
$$

Since $\Gamma$ and $Z^{-1}$ differ in their coupling constants, $\Gamma_{R}$ cannot be formed by simple multiplication. There are two steps involved. First, couplingconstant renormalization is carried out by substituting the bare coupling constant

$g^{2}(M)=g^{2}(\mu)\left[1+g^{2}(\mu) b_{1} \ln \frac{M^{2}}{\mu^{2}}+\cdots\right]$

into $\Gamma(p, M, g(M))$ leaving $\widetilde{\Gamma}(p, M, \mu, g(\mu))$. Equation (2.1) now reads

$$
\begin{aligned}
\Gamma_{R}(p, \mu, g(\mu))= & Z^{-1}(M, \mu, g(\mu)) \\
& \times \widetilde{\Gamma}(p, M, \mu, g(\mu)) .
\end{aligned}
$$

In $\widetilde{\Gamma}$ all overlapping divergences with respect to coupling-constant renormalization (self-energy and vertices) are consequently removed. The second step consists in wave-function renormalization. Multiplication of $Z^{-1}$ with $\widetilde{\Gamma}$ removes all remaining overlapping divergences plus the local UV counterterms.

Mass factorization in DI scattering is expressed by

$$
\begin{aligned}
\mathcal{S}(q, \mu, g(\mu))= & A^{-1}(p, \mu, g(\mu)) \\
& \times \sigma(q, p, \mu, g(\mu)),
\end{aligned}
$$

where $\Subset$ is the Wilson coefficient function, $A$ is the reduced operator matrix element, and $\sigma$ stands for the DI parton cross section (structure functions). Note all moment indices are ignored throughout the paper. Comparing Eqs. (2.3) and (2.4), we observe an analogy between multiplicative renormalization of the Green's function $\widetilde{\Gamma}$ in Eq. (2.3) and mass factorization of the DI cross section $\sigma$ in Eq. (2.4). The role of the UV singularity $M$ is taken over by the mass singularity $p^{2}$ whereas the renormalization constant $Z^{-1}$ is replaced by the inverse of the operator matrix element (OME) $A^{-1}$. Similarly, the renormalized Green's function $\Gamma_{R}$ is replaced by the Wilson coefficient $\Subset$. All diagrams contributing to $A^{-1}$ serve as mass counterterms for the mass-singular DI cross section $\sigma$. Since all mass singularities are universal, one infers that the initial-state mass singularities are removed from the cross section by the same $A^{-1}$ irrespective of which process one considers. Mass factorization proceeds in one step only, since there is no mass analog to coupling-constant renormalization. One 
observes a further difference: an $n$-point Green's function $\Gamma^{(n)}$ is superficially UV divergent or UV convergent, but not both. Cross sections, however, get contributions from mass-finite as well as from mass-singular graphs.

Mass factorization of the DY parton cross section $W$ implies that the DY analog of the Wilson coefficient

$$
\widetilde{C}^{\mathrm{DY}}(Q, \mu, g(\mu))=\left[A\left(p_{1}, \mu, g(\mu)\right) A\left(p_{2}, \mu, g(\mu)\right)\right]^{-1} W\left(Q, p_{1}, p_{2}, \mu, g(\mu)\right)
$$

is mass finite. From the universality of mass singularities it follows that the $A\left(p_{i}, \mu, g(\mu)\right)$ in Eq. (2.5) can be identified with the operator matrix elements of DI scattering.

Let us point out a new aspect here: $W\left(Q, p_{1}, p_{2}\right)$ reveals mass singularities from both incoming lines which can be traced back to the 2PR graphs in the sense of Ref. 2(c). Mass factorization allows the general form of the DY parton cross section

$$
W\left(Q, p_{1}, p_{2}, \mu, g(\mu)\right)=\sigma\left(Q, p_{1}, \mu, g(\mu)\right) \sigma\left(Q, p_{2}, \mu, g(\mu)\right) \Delta(1, \bar{g}),
$$

where $\sigma$ stands for the DI parton cross section (usually structure function $\mathscr{F}_{2}$ ), and the DY correction term $\Delta(1, \bar{g})$ is expanded in the running coupling constant $\bar{g}$ (and for later purposes in $g^{2}$ ) giving

$$
\Delta=\left(1+\bar{g}^{2} \Delta_{1}+\bar{g}^{4} \Delta_{2}+\cdots\right)=\left[1+g^{2} \Delta_{1}+g^{4}\left(\Delta_{2}+b_{1} \Delta_{1} \ln \frac{Q^{2}}{\mu^{2}}\right)+\cdots\right] .
$$

The coefficient $b_{1}$ follows from the expansion of the $\beta$ function

$$
\beta(g)=b_{1} g^{3}+b_{2} g^{5}+\cdots .
$$

\section{MASS FACTORIZATION WITH THE BPHZ METHOD}

In this section, we apply the BPHZ method, similar to renormalization in usual perturbation theory, on mass factorization. From the analogy exposed in the previous section, we learn that all diagrams contributing to $A^{-1}\left[\left(A_{1} A_{2}\right)^{-1}\right]$ serve as mass counterterms for the mass-singular DI (DY) parton cross sections. The BPHZ method gives an elegant and systematic way to determine the counterterms of each cut graph and allows for an easy formation of the mass-factorized cross sections.

The BPHZ renormalization of the usual Feynman diagrams is well known. ${ }^{6}$ It is carried out in two steps: first the overlapping divergences, consisting of UV-singular times UV-finite contributions, are disposed of via Bogoliubov's $R$ operation. In the second step, the remaining UV singularities are removed by the local counterterms.

\section{A. Classification of mass singularities}

In renormalization, all UV-divergent graphs are first classified according to their UV characteris- tics. We proceed here in a similar way. Before going to the formal steps of mass factorization, we analyze and classify the cut graphs according to their mass-singular characteristics. We consider graphs with initial-state mass singularities only (which will be mass factorized) since all final-state mass singularities, due to the Kinoshita-LeeNauenberg (KLN) theorem, ${ }^{9}$ cancel. In Ref. 2(c) a systematic method based on dimensional analysis was introduced. In $\left(\Phi^{3}\right)_{6}$ theory one finds the simple rule: a graph is only mass singular if there is a pair of equal propagators, and every additional pair introduces new mass singularities which appear via higher powers in $\ln p^{2}$. In analogy to the terminology used in $\mathrm{BPHZ}$ renormalization, we therefore split the cut graphs into several groups.

(a) Graphs which are mass singular and give rise to new counterterms. They are subdivided into the following classes: $\left(a_{1}\right)$ primitively divergent graphs, $\left(a_{2}\right)$ mass-divergent graphs which have no internal self-energy and strong-vertex correction, and $\left(a_{3}\right)$ mass-divergent graphs which contain internal self-energy and strong-vertex corrections.

(b) Mass-finite graphs.

(c) Mass-singular graphs contributing no new counterterms meaning that one of the subgraphs belongs to class (b).

We add a few remarks on the graphs in classes (a). Those belonging to class $\left(a_{1}\right)$ have only one pair of equal propagators and behave like $\ln p^{2}$ as $p^{2} \rightarrow 0$. The class $\left(\mathrm{a}_{2}\right)$ graphs with $n$ loops $(n$ rungs) reveal $\left(\ln p^{2}\right)^{n}$ as $p^{2} \rightarrow 0$. The general form 
of the DI cross section, built up by $\left(a_{2}\right)$ graphs only, therefore reads

$$
\sigma^{(n)}=\left(g^{2}\right)^{n} \sum_{i=0}^{n} f_{i} \ln ^{i} \frac{q^{2}}{p^{2}} .
$$

Equation (3.1) follows from dimensional arguments (only $p^{2}, q^{2}$ are available) and the observation that $\sigma^{(n)}$ is derived from $\sigma^{(n-1)}$ via

$$
\sigma^{(n)}=g^{2} \int_{p^{2}}^{q^{2}} \frac{d p^{\prime 2}}{p^{\prime 2}} \sigma^{(n-1)},
$$

where $d p^{\prime 2} / p^{\prime 2}$ represents the angular integration leading to the mass-singular behavior of $\sigma^{(n)}$ since

$$
\int_{p^{2}}^{q^{2}} \frac{d p^{\prime 2}}{p^{\prime 2}}\left[\ln \frac{q^{2}}{p^{\prime 2}}\right]^{i}=\frac{1}{i+1} \ln ^{i+1} \frac{q^{2}}{p^{2}} .
$$

Equation (3.1) follows. Mass factorization of Eq. (3.1) requires the separation $\ln \left(q^{2} / p^{2}\right)$ $=\ln \left(q^{2} / \mu^{2}\right)+\ln \left(\mu^{2} / p^{2}\right)$ which leads to mass-finite times mass-singular terms as $\ln ^{i}\left(q^{2} / \mu^{2}\right) \ln ^{j}\left(\mu^{2} / p^{2}\right)$ which can be cast in the form $\ln ^{i}\left(q^{2} / p^{2}\right) \ln ^{j}\left(\mu^{2} / p^{2}\right)$. Mass-singular contributions of this latter form are (in the terminology of 't Hooft and Veltman ${ }^{7}$ ) called overlapping divergences. Their removal is in mass factorization the principal difficulty. The reduced parton cross section is subsequently written as $\widetilde{\sigma}_{1}\left(q^{2} / \mu^{2}\right)+\widetilde{\sigma}_{2}\left(\mu^{2} / p^{2}\right)$. Subtraction of the last term, i.e., $\widetilde{\sigma}_{2}\left(\mu^{2} / p^{2}\right)$ (still by mass factorization), leads finally to the Wilson coefficient function. Diagrams of class $\left(a_{3}\right)$ are generated by inserting self-energies and strong vertices in the lower-order graphs of classes $\left(a_{1}\right)$ and $\left(a_{2}\right)$. Suppose the isolated vertex and/or self-energy behaves as $\left(\ln p^{2}\right)^{i}$ as $p^{2} \rightarrow 0$. Their insertion in a cut graph then raises the power of its leading mass singularity just by $i$. The insertion of strong vertices/self-energies in the $\left(a_{1}\right)$ and $\left(a_{2}\right)$ graphs does not lead to additional overlapping divergences. The argument is simple: insert $\ln ^{i}\left(\mu^{2} / p^{2}\right)$ in a graph of $\left(a_{1}\right)$. The new graph then behaves as

$$
\begin{aligned}
\int_{p^{2}}^{q^{2}} \frac{d p^{\prime 2}}{p^{\prime 2}} \ln ^{i} \frac{\mu^{2}}{p^{\prime 2}} & \\
& =\frac{-1}{i+1}\left(\ln i+1 \frac{q^{2}}{\mu^{2}}-\ln ^{i+1} \frac{\mu^{2}}{p^{2}}\right)
\end{aligned}
$$

with $\ln ^{i+1}\left(q^{2} / p^{2}\right)$ and $\ln ^{i+1}\left(\mu^{2} / p^{2}\right)$ already separated. Consequently, no extra subtraction of overlapping divergences has to be carried out. The same arguments apply if self-energy and vertex corrections are inserted in the graphs of class $\left(a_{2}\right)$.

We now focus our interest on mass factorization which, for an arbitrary DI cut graph, proceeds in the BPHZ method along similar steps as in renormalization. Our presentation will be limited to the graphs in class (a) (giving rise to new counterterms) which expose all difficulties. Extension to the other classes then becomes trivial.

\section{B. Taylor expansion, $\boldsymbol{R}$ operation, forest formula}

Following closely the work of Zimmermann on BPHZ renormalization, ${ }^{6}$ we present here the analogous definitions and arguments for mass factorization. In the subsequent subsections, we discuss some DI/DY nonsinglet graphs as illustrative examples.

Let us consider the graphs of class $\left(a_{1}\right)$ which are primitively divergent. $\sigma_{\Gamma}\left(p^{2}, q^{2}\right)$ denotes the cross section of a specific graph $\Gamma$ of order $\left(g^{2}\right)^{n}$. Its mass- subtraction term follows from a Taylor expansion around $q^{2}=\mu^{2}$, in formal notation $\left(-t^{\Gamma}\right) \sigma_{\Gamma}$, which is identified with $A^{-1}$. As in renormalization, $\left(-t^{\Gamma}\right)$ stands for the Taylor expansion in an external dynamical variable (e.g., $q^{2}$, but not $p^{2}, m^{2}, \lambda^{2}, \ldots$, since they are mass regulators). $\Gamma$ indicates the maximal number of derivatives taken into consideration which, however, shall not surmount the degree of divergence of the particular cut graph. Since mass divergences are only of logarithmic type, their degree of divergence is $d_{\Gamma}=0$. The operator matrix element $A_{\Gamma}$ follows from graph $\Gamma$ by contracting the two electromagnetic vertices to one point. It is also of order $\left(g^{2}\right)^{n}$ and primitively divergent which allows for the relation $A_{\Gamma}{ }^{-1}=-A_{\Gamma}$. As a result, the mass-factorized $\Gamma$ cross section (involving primitively divergent cut graphs only) reads

$$
{ }^{\varepsilon_{\Gamma}}=\left(1-t^{\Gamma}\right) \sigma_{\Gamma} \text {. }
$$

For DI scattering $\S_{\Gamma}$ is identified with the Wilson coefficient.

We consider the graphs of classes $\left(a_{2}\right)$ and $\left(a_{3}\right)$. Let us first give some definitions. Any graph $\Gamma$ in these classes can be broken into mass-singular subgraphs $\left\{\gamma_{1} \cdots \gamma_{k} \cdots\right\}$; they are connected and 1PI (proper). In analogy to the "renormalization parts," in renormalization theory, the $\gamma_{i}$ are called mass-factorization parts. We define the reduced diagram. Let $\left\{\gamma_{1} \cdots \gamma_{c}\right\}$ be a set of disjoint massfactorization parts meaning $\gamma_{i} \cap \gamma_{j}=\varnothing$ for $i \neq j$ and $\gamma_{i} \neq \Gamma$. The reduced diagram

$$
F=\Gamma /\left\{\gamma_{1} \ldots \gamma_{c}\right\}
$$


is defined by contracting each $\gamma_{i}$ in the set $\left\{\gamma_{1} \cdots \gamma_{c}\right\}$ to one point.

The graphs of classes $\left(a_{2}\right)$ and $\left(a_{3}\right)$ give rise to overlapping divergences (in the sense of 't Hooft and Veltman ${ }^{7}$ ) which are removed by Bogoliubov's $R$ operation. The reduced cross section $\bar{\sigma}_{\Gamma}$ has all these overlapping divergences removed; it is recursively defined by

$\bar{\sigma}_{\Gamma}=\sigma_{\Gamma}+\sum_{F} \sigma_{F} \prod_{\tau=1}^{c}\left(-t^{\gamma_{\tau}}\right) \bar{\sigma}_{\gamma_{\tau}} \quad(R$ operation $)$.

The sum extends over all sets of disjoint mass factorization parts of $\Gamma$. The final overall subtraction is carried out by

$$
\left(-t^{\Gamma}\right) \bar{\sigma}_{\Gamma} \equiv A_{\Gamma}{ }^{-1} \text {. }
$$

Since in the $\left(\mathrm{a}_{2}\right)$ and $\left(\mathrm{a}_{3}\right)$ classes $\Gamma$ is not primitively divergent, we may not identify $A_{\Gamma}{ }^{-1}=-A_{\Gamma}$. Formation of $A_{\Gamma}{ }^{-1}$ leads here to a combination of lower-order matrix elements corresponding to the subgraphs of $\Gamma$. As for the $\left(a_{1}\right)$ class, however, we may set

$$
\left(-t^{\Gamma}\right) \sigma_{\Gamma} \equiv-A_{\Gamma}
$$

since the Taylor expansion of $\sigma_{\Gamma}$ leads directly to the operator matrix element which is obtained by contracting the electromagnetic vertices to one point. Since there are only logarithmic, but no linear or even more divergent, mass divergences the lowest-order term in the Taylor expansion is only needed. After the overall divergences $\left(-t^{\Gamma}\right) \bar{\sigma}_{\Gamma}$ have been removed, the remaining Wilson coefficient is given by

$$
\varsigma_{\Gamma}=\left(1-t^{\Gamma}\right) \bar{\sigma}_{\Gamma} \text {. }
$$

Applying Hepp's renormalization-theory arguments $^{6 \mathrm{c}}$ in an analogous way to mass factorization, one can show that the above procedure, as outlined from Eqs. (3.6) to (3.9), renders a massfinite parton cross section $\mathbb{E}_{\Gamma}$. In DI scattering, it is identified with the Wilson coefficient. After rearranging terms, it is finally cast in the forest formula

$$
\mathfrak{\mho}_{\Gamma}=\sum_{U} \prod_{\lambda \in U}\left(-t^{\lambda}\right) \sigma_{\Gamma} \text {. }
$$

The sum extends over all possible $\Gamma$ forests of $U$. $U$ is a set of nonoverlapping mass-factorization parts of $\Gamma$ which includes $\Gamma$ and the empty set $\varnothing$. $-t^{\lambda} \sigma_{\Gamma}$ removes the $\lambda$ subgraph from $\Gamma$ and replaces it by the counterterm $A_{\lambda}{ }^{-1} \equiv\left(-t^{\lambda}\right) \bar{\sigma}_{\lambda}$. Following Ref. 6(b), we define the BPHZ overlapping (mass) divergences. Two mass-factorization parts are said to overlap if none of the three following conditions holds true: $\gamma_{1} \subset \gamma_{2}, \gamma_{2} \subset \gamma_{1}, \gamma_{1} \cap \gamma_{2}=\varnothing$. In DI scattering there are no overlapping BPHZ divergences. In this case, the $\gamma_{i}$ are strongly ordered, i.e., $\gamma_{1} \subset \gamma_{2} \subset \cdots \subset \gamma_{n} \equiv \Gamma$. Therefore, Eqs. (3.7) and (3.10) can be simplified to

$$
\begin{aligned}
& \bar{\sigma}_{\Gamma}=\sigma_{\Gamma}+\sum_{\gamma_{i}} \sigma_{\Gamma / \gamma_{i}}\left(-t^{\gamma_{i}}\right) \bar{\sigma}_{\gamma_{i}}, \\
& \varsigma_{\Gamma}=\prod_{\gamma}\left(1-t^{\gamma}\right) \sigma_{\Gamma},
\end{aligned}
$$

with $\gamma$ representing all mass-factorization parts of $\Gamma$ including $\Gamma$ and $\varnothing$.

All graphs of class (b) do not need any subtraction: $\mathbb{C}_{\Gamma}=\sigma_{\Gamma}$. The counterterms of class (c) were already determined while discussing the class (a) graphs above. The overall counterterm of this class (c) follows immediately from a contraction of the two electromagnetic vertices (including their strong corrections and internal self-energies) to one point.

We extend our reasoning to the DY process where both incoming lines (called 1 and 2) give rise to mass singularities. We proceed as in DI scattering where each DY cut graph $\Gamma$ is broken into disjoint mass-factorization parts $\gamma_{\tau} \subset \Gamma$. Let $\Gamma_{i}$ $(i=1,2)$ be the maximal mass-factorization part containing the mass singularities of one state $p_{i}$ only. It reveals the topology of the DI cut graphs and therefore the same mass singularities are found. This is the deeper reason why the operator matrix elements can be used as counterterms for the DY graphs. In contrast to DI scattering, the DY graphs give rise to BPHZ overlapping divergences. In particular, $\Gamma_{1}$ and $\Gamma_{2}$ are overlapping. Mass factorization proceeds in an analogous way as in DI scattering. The DY coefficient function is derived from Eqs. (3.7) and (3.10). Since there are two sources of mass singularities, each originating from one of the initial-state lines, the counterterms of the DY cross section contribute to $\left[A\left(p_{1}\right) A\left(p_{2}\right)\right]^{-1}$.

In analogy to DI scattering, the individual DY cut graphs $W_{\Gamma}$ are mass factorized by counterterms which satisfy (see Sec. VII)

$$
\left(-t^{\Gamma}\right) W_{\Gamma}^{(n)}=-\left.A_{\Gamma_{1}}^{(k)} A_{\Gamma_{2}}^{(n-k)}\right|_{\Gamma_{1}, \Gamma_{2} \subset \Gamma}, \quad\left(-t^{\Gamma}\right) \bar{W}_{\Gamma}^{(n)}=\left.\left[A_{\Gamma_{1}}^{(k)} A_{\Gamma_{2}}^{(n-k)}\right]^{-1}\right|_{\Gamma_{1}, \Gamma_{2} \subset \Gamma} .
$$




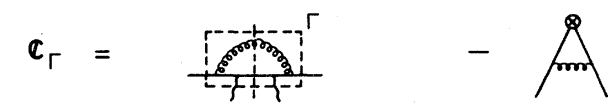

FIG. 1. DI graph of class $\left(a_{1}\right)$.

The forest formula for the DY process is the same as in DI scattering [see Eq. (3.11)]. After some manipulations the $D Y$ forest formula can be cast into

$$
\mho_{\Gamma}^{D Y}=\left[\prod_{\gamma \in \Gamma_{1}}\left(1-t^{\gamma}\right)\right]\left[\prod_{\lambda \in \Gamma_{2}}\left(1-t^{\lambda}\right)\right] W_{\Gamma}^{\mathrm{DY}} .
$$

The products extend over all mass-factorization parts of $\Gamma$ which belong either to $\gamma_{1}$ or $\gamma_{2}$. Therefore, all mass-factorization parts $\gamma, \lambda$ which contain $p_{1}$ as well as $p_{2}$ singularities will be excluded from the two products.

\section{DI examples (nonsinglet)}

We explain the above general insight by explicit examples in DI scattering which stand representa- tive for the classes $\left(a_{1}\right)-\left(a_{3}\right)$. Our main aim is to demonstrate how the mass-factorized contribution of each graph to the Wilson coefficient is derived.

We start with the primitively divergent cut graph exposed in Fig. 1 [of class $\left(a_{1}\right)$ ]. The cross section is denoted by $\sigma_{\Gamma}$ and its counterterm reads $-t^{\Gamma} \sigma_{\Gamma}=-A_{\Gamma} \sigma_{0} . \sigma_{0}(\equiv 1)$ is the naive parton cross section without any strong-interaction corrections. The Wilson coefficient is given by

$$
\mathfrak{夭}_{\Gamma}=\sigma_{\Gamma}-A_{\Gamma}
$$

We consider the class $\left(a_{2}\right)$ cut graph shown in Fig. 2 which again is denoted by $\Gamma$ with its cross section $\sigma_{\Gamma}$; it gives rise to overlapping divergences. In order to carry out the $R$ operation, we have in Fig. 2(a) indicated the mass-factorization parts $\gamma_{1}, \gamma_{2}$. The two reduced diagrams then follow from successively eliminating the innermost gluon line $\left(\rightarrow \Gamma / \gamma_{1}\right)$ and then the next following gluon line $\left(\rightarrow \Gamma / \gamma_{2}\right)$. The reduced parton cross section is recursively defined by the $R$ operation [Fig. 2(b)].

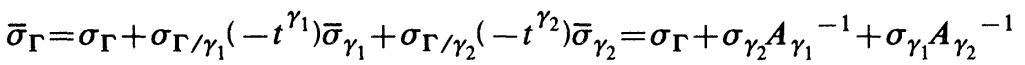

$$
\begin{aligned}
& =\sigma_{\Gamma}-\sigma_{\gamma_{2}} A_{\gamma_{1}}-\sigma_{\gamma_{1}}\left(A_{\gamma_{2}}-A_{\gamma_{1}} A_{\gamma_{1}}\right) \text {. }
\end{aligned}
$$

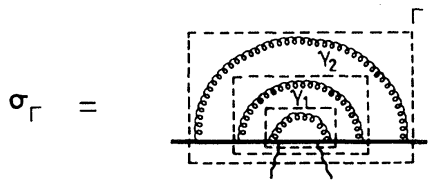

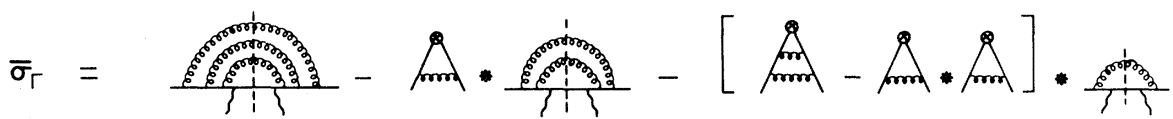

$$
\begin{aligned}
& \text { (b) }
\end{aligned}
$$

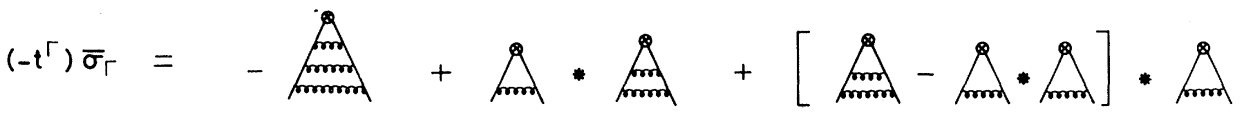

$$
\begin{aligned}
& \text { (c) }
\end{aligned}
$$

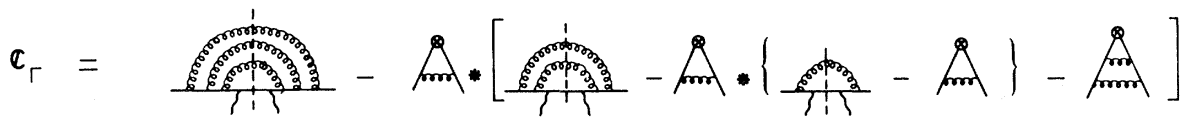

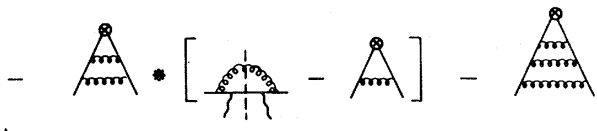

FIG. 2. DI graph of class $\left(a_{2}\right)$ : (a) mass-factorization parts, (b) $R$ operation, (c) overall subtraction, (d) Wilson coefficient. 


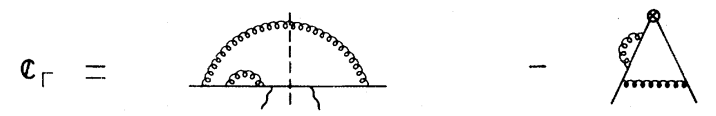

FIG. 3. DI graph of class $\left(a_{3}\right)$.

We have used the fact that $\gamma_{1}$ is primitively divergent: $A_{\gamma_{1}}{ }^{-1}=-A_{\gamma_{1}}$. In an analogous order- $\left(g^{2}\right)^{2}$ analysis, one easily gets

$$
\left(-t^{\gamma_{2}}\right) \bar{\sigma}_{\gamma_{2}}=\left(-A_{\gamma_{2}}+A_{\gamma_{1}} A_{\gamma_{1}}\right) .
$$

The overall subtraction is carried out as [Fig. 2(c)]

$$
\begin{aligned}
\left(-t^{\Gamma}\right) \bar{\sigma}_{\Gamma}= & \left(-t^{\Gamma}\right) \sigma_{\Gamma}-A_{\gamma_{1}}\left(-t^{\Gamma}\right) \sigma_{\gamma_{2}} \\
& -\left(A_{\gamma_{2}}-A_{\gamma_{1}} A_{\gamma_{1}}\right)\left(-t^{\Gamma}\right) \sigma_{\gamma_{1}} \\
= & -A_{\Gamma}+A_{\gamma_{1}} A_{\gamma_{2}}+\left(A_{\gamma_{2}}-A_{\gamma_{1}} A_{\gamma_{1}}\right) A_{\gamma_{1}} \\
\equiv & A_{\Gamma}{ }^{-1}
\end{aligned}
$$

where we used according to the definition $\left(-t^{\Gamma}\right) \sigma_{\gamma_{1}}$ $=-A_{\gamma_{1}}$. Determination of the Wilson coefficient is now easy [Fig. 2(d)]:

$$
\begin{aligned}
C_{\Gamma}= & \left(1-t^{\Gamma}\right) \bar{\sigma}_{\Gamma} \\
= & \sigma_{\Gamma}-A_{\gamma_{1}}\left[\sigma_{\gamma_{2}}-A_{\gamma_{1}}\left(\sigma_{\gamma_{1}}-A_{\gamma_{1}}\right)-A_{\gamma_{2}}\right] \\
& -A_{\gamma_{2}}\left(\sigma_{\gamma_{1}}-A_{\gamma_{1}}\right)-A_{\Gamma} .
\end{aligned}
$$

In Fig. 3, we discuss an example for the $\left(a_{3}\right)$ class of graphs with its cross section denoted by
$\sigma_{\Gamma_{0}}$. Note that the subgraph with the self-energy is not $1 \mathrm{PI}$ and that it contains no mass-factorization parts. The chain of reasoning for Fig. 1 applies here in the same way leaving

$$
C_{\Gamma_{0}}=\sigma_{\Gamma_{0}}-A_{\Gamma_{0}}
$$

as indicated by Fig. 3. A more complicated graph, still in the $\left(a_{3}\right)$ class, is shown in Fig. 4 with the complete graph denoted by $\Gamma$, the indicated massfactorization part $\gamma_{1}$, and the reduced diagram $\Gamma / \gamma_{1} \equiv \Gamma_{0}$ [Fig. 4(a)] being the one just considered above. The $R$ operation reads [Fig. 4(b)]

$$
\begin{aligned}
\bar{\sigma}_{\Gamma} & =\sigma_{\Gamma}+\sigma_{\Gamma / \gamma_{1}}\left(-t^{\gamma_{1}}\right) \bar{\sigma}_{\gamma_{1}} \\
& =\sigma_{\Gamma}+\sigma_{\Gamma_{0}} A_{\gamma_{1}}{ }^{-1} \\
& =\sigma_{\Gamma}-\sigma_{\Gamma_{0}} A_{\gamma_{1}}
\end{aligned}
$$

with the overall subtraction being carried out as [Fig. 4(c)]

$$
\begin{aligned}
\left(-t^{\Gamma}\right) \bar{\sigma}_{\Gamma} & =\left(-t^{\Gamma}\right) \sigma_{\Gamma}-A_{\gamma_{1}}\left(-t^{\Gamma}\right) \sigma_{\Gamma_{0}} \\
& =-A_{\Gamma}+A_{\gamma_{1}} A_{\Gamma_{0}} \equiv A_{\Gamma}{ }^{-1} .
\end{aligned}
$$

The Wilson coefficient is readily derived leaving [Fig. 4(d)]

$$
\begin{aligned}
\mathfrak{夭}_{\Gamma} & =\left(1-t^{\Gamma}\right) \bar{\sigma}_{\Gamma} \\
& =\sigma_{\Gamma}-A_{\gamma_{1}}\left(\sigma_{\Gamma_{0}}-A_{\Gamma_{0}}\right)-A_{\Gamma} .
\end{aligned}
$$

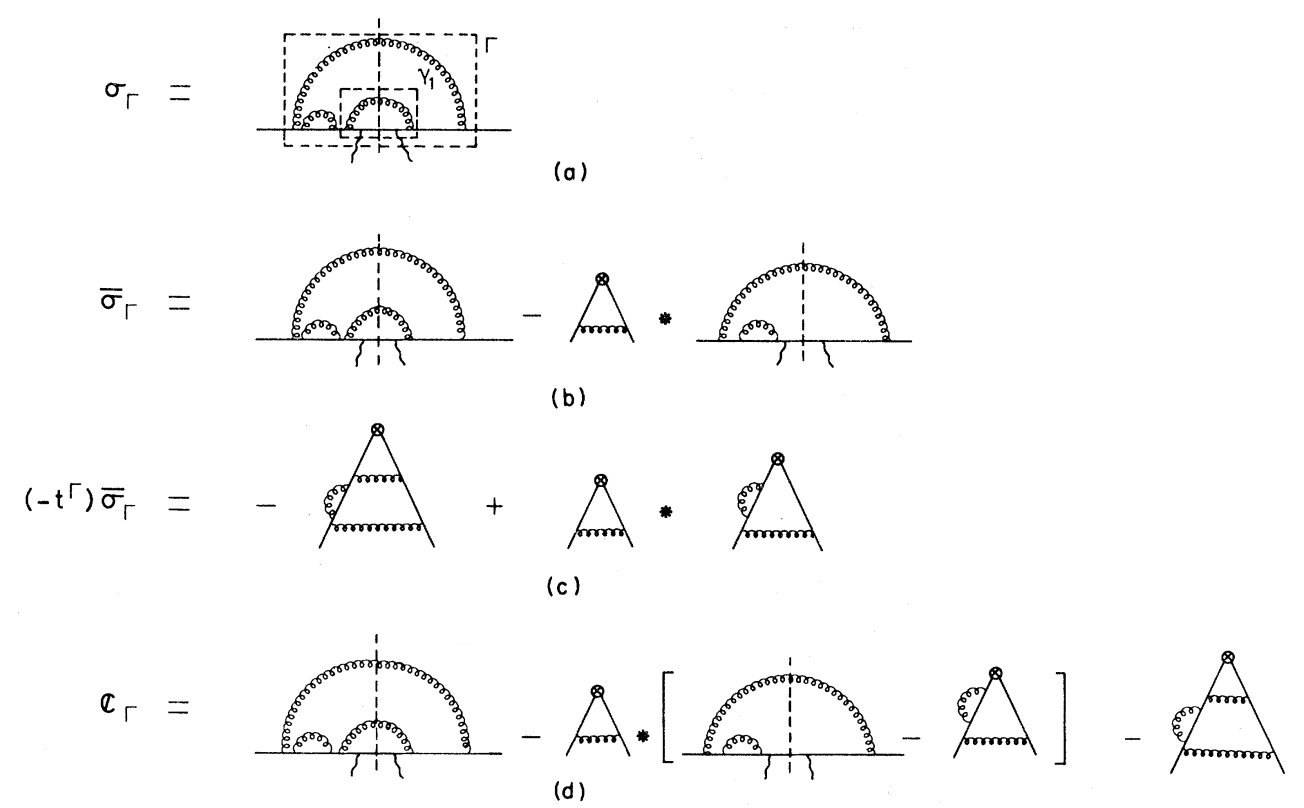

FIG. 4. DI graph of class $\left(\mathrm{a}_{4}\right)$ : (a) mass-factorization parts, (b) $R$ operation, (c) overall subtraction, (d) Wilson coefficient. 

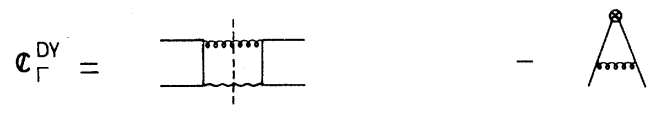

FIG. 5. DY graph of class $\left(a_{1}\right)$.

D. DY examples (nonsinglet)

We extend our illustration of the general presentation of the theory in Sec. III B to the DY process. The cut graph in Fig. 5 is primitively divergent and therefore stands representative for class $\left(a_{1}\right)$. Since there are no mass-factorization parts, it is treated as Fig. 1 in DI scattering. $W_{\Gamma}$ denotes its cross section and Eq. (3.13) leads to

$$
\left(-t^{\Gamma}\right) W_{\Gamma}=-A_{\Gamma} A_{\phi}=-A_{\Gamma}
$$

since $A_{\phi}=1$. The DY coefficient function follows (Fig. 5):

$$
\text { (厄) } \stackrel{\mathrm{DY}}{\Gamma}=W_{\Gamma}-A_{\Gamma} \text {. }
$$

The slightly more complicated example in Fig. 6 reveals overlapping divergences; it stands representative for class $\left(a_{2}\right)$. The cut graph is denoted by
$\Gamma$, and we have indicated the mass-factorization part $\gamma_{1}$ as well as the maximal mass-factorization part $\Gamma_{1}$ with respect to line $p_{1}$. The maximal mass-factorization part with respect to line $p_{2}$ is empty: $\Gamma_{2}=\varnothing$. All steps now proceed as in DI scattering:

$$
\begin{aligned}
& \bar{W}_{\Gamma}=W_{\Gamma}-W_{\gamma_{1}} A_{\gamma_{1}}, \\
& \left(-t^{\Gamma}\right) \bar{W}_{\Gamma}=\left(-A_{\Gamma}+A_{\gamma_{1}} A_{\gamma_{1}}\right),
\end{aligned}
$$

leaving the DY coefficient

$$
\begin{aligned}
{ }_{\Gamma}^{\mathrm{DY}} & =\left(1-t^{\Gamma}\right) W_{\Gamma} \\
& =W_{\Gamma}-A_{\gamma_{1}}\left(W_{\gamma_{1}}-A_{\gamma_{1}}\right)-A_{\Gamma} .
\end{aligned}
$$

By Fig. 7, we demonstrate the complexity of a complicated example, still of class $\left(a_{2}\right)$. The (maximal) mass-factorization part of the line $p_{1}$ is $\left(\Gamma_{1}\right) \gamma_{1}$; similarly, the maximal part of line $p_{2}$ is denoted by $\Gamma_{2} . \quad \gamma_{2}$ is a factorization part involving mass singularities of both incoming lines, and $\Gamma$ denotes the complete cut graph [Fig. 7(a)]. The $R$ operation leads to [see Fig. 7(b)]

$$
\begin{aligned}
\bar{W}_{\Gamma} & =W_{\Gamma}+W_{\Gamma / \Gamma_{1}}\left(-t^{\Gamma_{1}}\right) \bar{W}_{\Gamma_{1}}+W_{\Gamma / \Gamma_{2}}\left(-t^{\Gamma_{2}}\right) \bar{W}_{\Gamma_{2}}+W_{\Gamma / \gamma_{1}}\left(-t^{\gamma_{1}}\right) \bar{W}_{\gamma_{1}}+W_{\Gamma / \gamma_{2}}\left(-t^{\gamma_{2}}\right) \bar{W}_{\gamma_{2}} \\
& =W_{\Gamma}-W_{\Gamma_{2}}\left(A_{\Gamma_{1}}-A_{\gamma_{1}} A_{\gamma_{1}}\right)-W_{\Gamma_{1}} A_{\Gamma_{2}}-W_{\gamma_{2}} A_{\gamma_{1}}+W_{\gamma_{1}} A_{\gamma_{1}} A_{\Gamma_{2}} .
\end{aligned}
$$

The operation $\left(-t^{\Gamma}\right)$ acting on Eq. (3.27) leads to the overall subtraction [Fig. 7(c)]. We discuss its effect on the individual terms. According to Eq. (3.13), we have

$$
\left(-t^{\Gamma}\right) W_{\Gamma}=-A_{\Gamma_{1}} A_{\Gamma_{2}},\left(-t^{\Gamma}\right) W_{\Gamma_{i}}=-A_{\Gamma_{i}}, \quad\left(-t^{\Gamma}\right) W_{\gamma_{2}}=-A_{\gamma_{1}} A_{\gamma_{2}} .
$$

In the last equation, $\gamma_{2}$ involves $p_{1}$ - and $p_{2}$-line singularities. Formation of the DY coefficient function now is trivial [Fig. 7(d)]; we find

$$
\begin{aligned}
\mathfrak{\complement}_{\Gamma}=\left(1-t^{\Gamma}\right) \bar{W}_{\Gamma}= & W_{\Gamma}-A_{\Gamma_{2}}\left[\sigma_{\Gamma_{1}}-A_{\gamma_{1}}\left(W_{\gamma_{1}}-A_{\gamma_{1}}\right)-A_{\Gamma_{1}}\right] \\
& -A_{\gamma_{1}}\left[W_{\gamma_{2}}-A_{\gamma_{1}}\left(W_{\Gamma_{2}}-A_{\Gamma_{2}}\right)-A_{\Gamma_{2}}\left(W_{\gamma_{1}}-A_{\gamma_{1}}\right)-A_{\gamma_{1}} A_{\Gamma_{2}}\right] \\
& \quad-A_{\Gamma_{1}}\left(W_{\Gamma_{2}}-A_{\Gamma_{2}}\right)-A_{\gamma_{1}} A_{\Gamma_{2}}\left(W_{\gamma_{1}}-A_{\gamma_{1}}\right)-A_{\Gamma_{1}} A_{\Gamma_{2}} \\
= & {\left[\left(1-t^{\Gamma_{1}}\right)\left(1-t^{\gamma_{1}}\right)\right]\left[\left(1-t^{\Gamma_{2}}\right)\right] W_{\Gamma} . }
\end{aligned}
$$

The last form can directly be derived from Eq. (3.14).

In Fig. 8, we have indicated class $\left(a_{3}\right)$ examples. Any more complicated structure involving self-

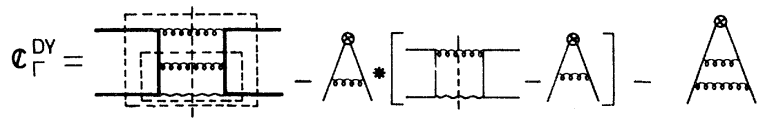

FIG. 6. DY graph of class $\left(a_{2}\right)$. energy and strong-vertex corrections can by analogous reasoning be deduced. Application of the above-demonstrated forest formalism applies in the same way.

\section{BPHZ MASS FACTORIZATION OF THE SINGLET GRAPHS}

We now extend our reasoning to the singlet graphs. BPHZ mass factorization for an individu- 


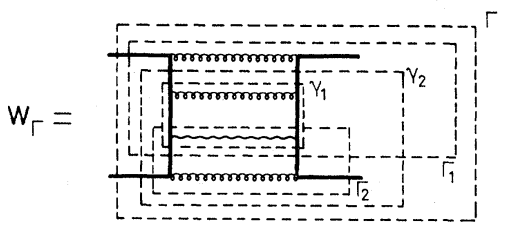

(a)

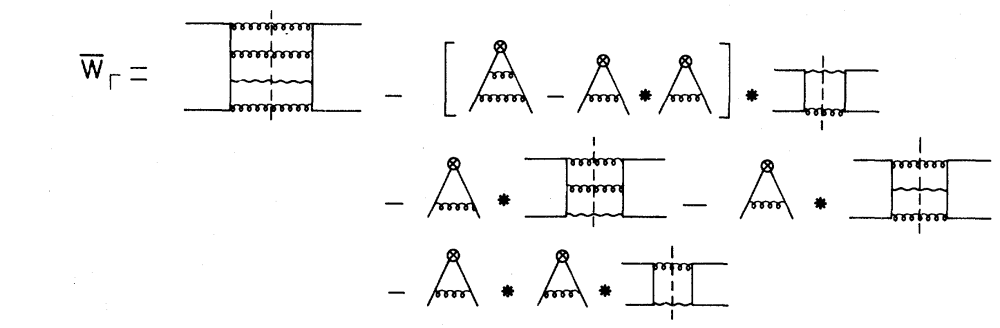

(b)

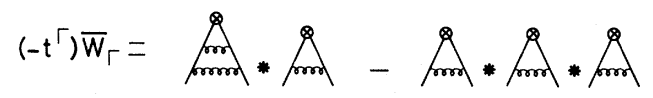

(c)

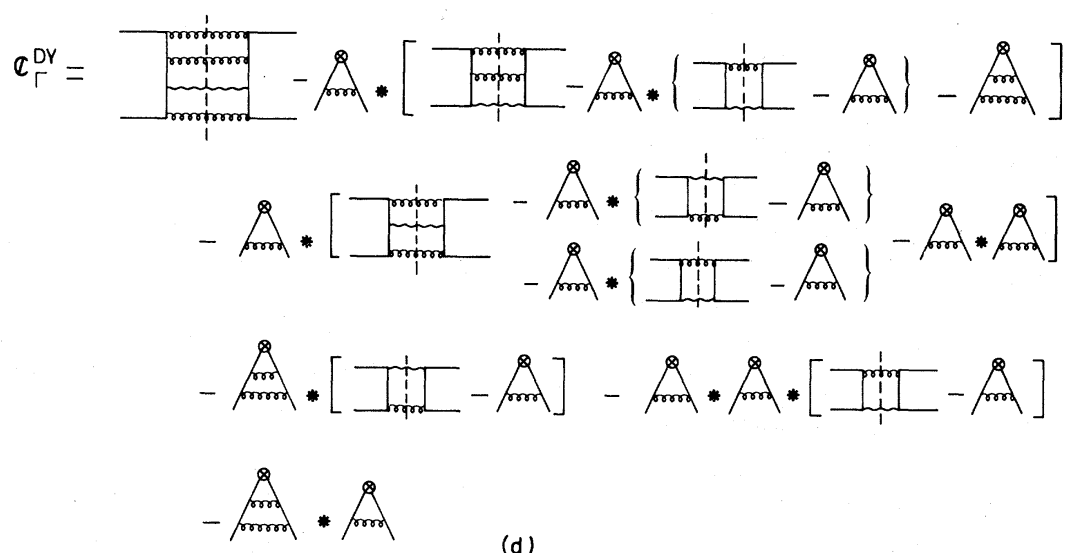

(d)

FIG. 7. DY graph of class $\left(\mathrm{a}_{2}\right)$ : (a) mass-factorization parts, (b) $R$ operation, (c) overall subtraction, (d) Wilson coefficient.

al DI/DY cut graph proceeds in spirit as explained above with slight modifications of the rules. While seeking mass factorization of the singlet cross sections, one comes across the phenomenon of operator mixing which implies that the OME is

represented by a matrix. We somewhat relax our

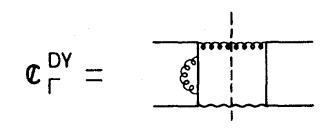

(a)
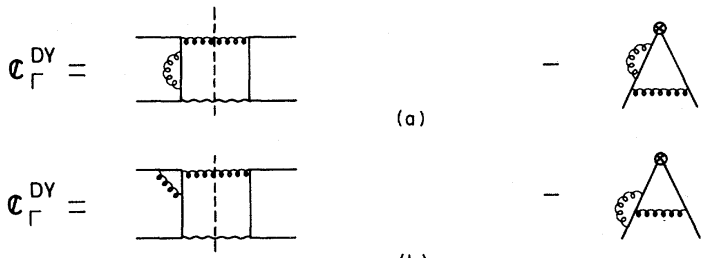

(b)

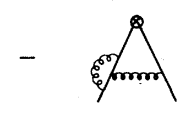

FIG. 8. DY graphs of class $\left(a_{3}\right)$ : (a) internal selfenergy, (b) internal strong-vertex correction. earlier arguments and now suppose a field theory with two different quanta, e.g., quarks $(q)$ and gluons $(g)$. We, however, still ignore all further gauge-theory complications such as IR divergences, gauge dependence of the individual cut graphs, and others (pseudoscalar fermion theory is a possible example). The inclusion of all such complications is postponed to Sec. VI.

\section{A. The changes}

In DI scattering the operator matrix element is now described by the matrix

$$
\mathfrak{A}=\left[\begin{array}{ll}
A^{q q} & A^{q \mathrm{~g}} \\
A^{g q} & A^{g \mathrm{~g}}
\end{array}\right] .
$$


Multiplicative renormalization in the case of mixing reads

$\mathfrak{2}_{R}(p, \mu, g(\mu))=3^{-1}(M, \mu, g(\mu)) \mathfrak{A}(p, M, g(M))$,

where $3^{-1}$ is now a matrix. If the external source is of weak or electromagnetic origin it only interacts with the quarks. In this case, the Wilson coefficient and the parton cross section become vectors. Mass factorization is cast in the form

$\overrightarrow{\widetilde{\mho}}(q, \mu, g(\mu))=\mathfrak{U}^{-1}(p, \mu, g(\mu)) \vec{\sigma}(q, p, \mu, g(\mu))$

with

$$
\overrightarrow{\mathfrak{\Im}}=\left(\begin{array}{l}
\Im q \\
\S g
\end{array}\right), \quad \vec{\sigma}=\left(\begin{array}{l}
\sigma^{q} \\
\sigma^{g}
\end{array}\right) .
$$

Comparing Eqs. (4.2) and (4.3) we observe that the simple analogy between multiplicative renormalization and mass factorization no longer holds true. $\overrightarrow{\widetilde{E}}$ and $\vec{\sigma}$ are vectors instead of matrices as in renormalization. This problem would not arise if the external source would couple to both quarks and gluons (one might think of it as being of gravitational ${ }^{10}$ origin or simply a scalar source). BPHZ mass factorization, however, still applies since the mass singularities are process independent and universal. Mass factorization of the DI virtualphoton cross section can still be carried out by subtracting operator matrix elements times DI cut graphs, the latter describing the interaction of the external scalar source with the gluons much in the same way as the off-shell photon with the quarks. We now make use of the fact that the Wilson coefficient is determined up to a finite operator renormalization; this allows replacement of some of the operator matrix elements by the corresponding virtual-photon DI cross sections and some of the scalar DI cross sections by their corresponding operator matrix element. This procedure leads finally to the Wilson coefficient as a product of OME's times virtual-photon DI cross sections much in the same way as in the nonsinglet case (see Sec. III).

In practice, we proceed as follows: Take a theory where the quarks interact with an electromagnetic source and the gluons interact with a (spurious) scalar source. ${ }^{10}$ The power-counting rules which determine the mass-singular characteristics of the cut graphs are the same as for the nonsinglet cut graphs. Hence, they are classified in groups (a), (b), and (c), as explained in Sec. III.

BPHZ mass factorization proceeds again via the usual $R$ operation which now, however, shall include the scalar-source-gluon interaction

$$
\bar{\sigma}_{\Gamma}=\sigma_{\Gamma}+\sum_{F} \sigma_{F}^{s}\left(-t^{\lambda}\right) \bar{\sigma}_{\lambda}+\sum_{F} \sigma_{F}\left(-t^{\lambda}\right) \bar{\sigma}_{\lambda} .
$$

All cross sections with the superscript $s$ involve the scalar-source-gluon coupling whereas all others are of the usual electromagnetic type. The Wilson coefficient follows from the familiar overall subtraction. In order to dispose in the Wilson coefficient of all terms containing the (unphysical) scalar source, we make use of the subtraction freedom while carrying out OME renormalization. Since it affects in the same way the Wilson coefficient we have the possibility to define a modified $R$ operation as

$$
\bar{\sigma}_{\Gamma}=\sigma_{\Gamma}+\sum_{F} \bar{\sigma}_{\lambda}\left(-t^{F}\right) \sigma_{F}^{s}+\sum_{F} \sigma_{F}\left(-t^{\lambda}\right) \bar{\sigma}_{\lambda} .
$$

Since the mass counterterms belonging to the DI cross section do not change apart from a finite renormalization they can be defined in the usual way. Mass factorization now proceeds as explained in the previous section

As in the nonsinglet case, the Wilson coefficient can be expressed by the forest formula [Eq. (3.10)]. On its right-hand side, we come across graphs involving the spurious scalar external source. Therefore, a modified forest formula is defined (as for the $R$ operation) by the replacement

$$
\sigma_{\Gamma / \gamma}^{s}\left(-t^{\lambda}\right) \bar{\sigma}_{\lambda} \Longrightarrow \bar{\sigma}_{\lambda}\left(-t^{\Gamma / \gamma}\right) \sigma_{\Gamma / \gamma}^{s} .
$$

The resulting Wilson coefficient is the same as in Eq. (3.10) apart from a finite renormalization. The replacement [Eq. (4.7)] is traced back to the modified $R$ operation rendering the (usual) Wilson coefficient up to a finite renormalization. The usual and modified $R$ operations remove both the overlapping divergences from $\sigma_{\Gamma}$ which is sufficient to prove mass factorization.

We indicate the changes for the DY process if singlet cut graphs are considered. Mass factorization now reads

$$
\mho_{k l}(Q, \mu, g(\mu))=\sum_{i, j}\left[\mathfrak{A}_{k i}\left(p_{1}, \mu, g(\mu)\right) \mathfrak{A}_{l j}\left(p_{2}, \mu, g(\mu)\right)\right]^{-1} W_{i j}\left(Q, p_{1}, p_{2}, \mu, g(\mu)\right),
$$



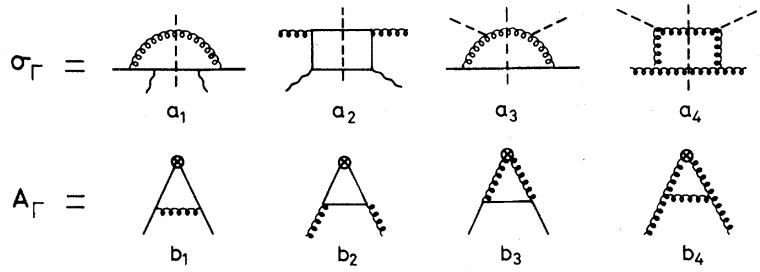

FIG. 9. Singlet DI scattering cross sections $a_{1}-a_{4}$, and the corresponding OME's $b_{1}-b_{4}$.

where $g \equiv g(\mu)$ and the indices $(i, j, k, l)=(q, g)$. As in the nonsinglet case, $\left[\mathfrak{A}\left(p_{1}\right) \mathfrak{H}\left(p_{2}\right)\right]$ provides the mass counterterms for the DY cross section.

BPHZ mass factorization proceeds in an analogous way as for the DI singlet cross sections, with a (spurious) scalar external source and the modified $R$ operation [Eq. (4.6)]. The counterterms, analogous to DI scattering, are defined as in the nonsinglet case [see Eq. (3.13)].

The above arguments apply also to the DY forest formula [Eq. (3.10)]. The DY forest formula, as exposed for nonsinglet graphs in Eq. (3.14), is carried over to the singlet case in unchanged form provided the scalar source-gluon interaction is admitted and the replacement [Eq. (4.7)] is used.

\section{B. DI examples (singlets)}

In Fig. 9 a set of primitively divergent cut graphs is exposed with the cross-section notation: $\sigma^{\gamma q}\left(\mathrm{a}_{1}\right), \sigma^{\gamma g}\left(\mathrm{a}_{2}\right), \sigma^{s q}\left(\mathrm{a}_{3}\right), \sigma^{s g}\left(\mathrm{a}_{4}\right)$. Similarly, we indicate in Fig. 9 the corresponding OME's which are formed via contraction of the external-source vertices (photon, scalar). The singlet OME's are denoted as $A^{q q}\left(\mathrm{~b}_{1}\right), A^{g q}\left(\mathrm{~b}_{2}\right), A^{q 8}\left(\mathrm{~b}_{3}\right), A^{g 8}\left(\mathrm{~b}_{4}\right)$ with the particular figure number indicated in brackets.

We illustrate our general reasoning by explicit examples in DI scattering. The contributions to the Wilson coefficient of the primitively divergent (singlet) graphs in Figs. $9\left(a_{2}\right)$ and $9\left(b_{2}\right)$ [class $\left(a_{1}\right)$ in Sec. III] are formed by

$$
\mathcal{C}_{\Gamma}^{g}=\sigma_{\Gamma}^{\gamma g}-A_{\Gamma}^{8 q} \text {. }
$$

We investigate the cut graph shown in Fig. 10 [of class $\left(a_{2}\right)$ with overlapping divergences] where our

$$
\left(-\tau^{r}\right) \bar{\sigma}_{\Gamma}=\sigma_{\Gamma}=\bar{\sigma}_{\Gamma=}
$$

FIG. 10. Singlet DI graph of class $\left(\mathrm{a}_{2}\right)$ : (a) mass factorization, (b) $R$ operation, (c) overall subtraction, (d) Wilson coefficient, (e) modified $R$ operation, (f) Wilson coefficient. 


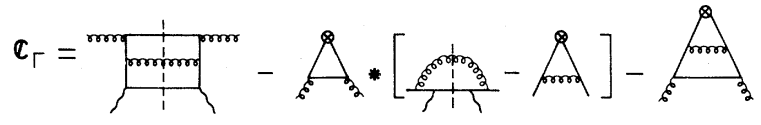

FIG. 11. Singlet DI graph of class $\left(a_{2}\right)$.

modification of the diagrammatic rule becomes transparent. We have indicated the massfactorization part $\gamma_{1} ; \Gamma$ denotes the complete graph [Fig. 10(a)]. The $R$ operation reads [Fig. 10(b)]

$$
\begin{aligned}
\bar{\sigma}_{\Gamma}^{\gamma q} & =\sigma_{\Gamma}^{\gamma q}+\sigma_{\Gamma / \gamma_{1}}^{s q}\left(-t^{\gamma_{1}}\right) \bar{\sigma}_{\gamma_{1}}^{\gamma g} \\
& =\sigma_{\Gamma}^{\gamma q}-\sigma_{\Gamma / \gamma_{1}}^{s q} A_{\gamma_{1}}^{g q} .
\end{aligned}
$$

Since the photon cannot couple to a gluon the (spurious) scalar external source comes into play. The overall subtraction follows from the Taylor expansion applied on the previous result together with Fig. 9:

$$
\left(-t^{\Gamma}\right) \bar{\sigma}_{\Gamma}^{\gamma q}=\left(-A_{\Gamma}^{q q}+A_{\Gamma / \gamma_{1}}^{q g}{ }_{\gamma_{1}}^{g q}\right)
$$

The Wilson coefficient trivially follows [Fig. 10(d)]:

$$
C_{\Gamma}^{q}=\sigma_{\Gamma}^{\gamma q}-A_{\gamma_{1}}^{g q}\left(\sigma_{\Gamma / \gamma_{1}}^{s q}-A_{\Gamma / \gamma_{1}}^{q g}\right)-A_{\Gamma}^{q q}
$$

This expression still contains the scalar external source. The form of the Wilson coefficient containing photon-quark interactions only follows from a finite renormalization of the OME and with it of the Wilson coefficient. We make use of the observation that the overlapping divergences appearing in the product: cross section (involving the scalar source) times OME does not change if $q^{2} \leftrightarrow \mu^{2}$ are interchanged. We therefore may set

$$
\sigma_{\Gamma / \gamma_{1}}^{s q} A_{\gamma_{1}}^{g q}=A_{\Gamma / \gamma_{1}}^{q g} \sigma_{\gamma_{1}}^{\gamma g}+O\left[\ln \frac{-q^{2}}{\mu^{2}}\right] .
$$

The last term $O\left(\ln q^{2} / \mu^{2}\right)$ changes the form of $\mathfrak{\complement}_{\Gamma}$ but only to a finite renormalization. Notice $A_{\Gamma / \gamma_{1}}^{q g}\left(A_{\gamma_{1}}^{g q}\right)$ is the counterterm of $\sigma_{\Gamma / \gamma_{1}}^{s q}\left(\sigma_{\gamma_{1}}^{\gamma g}\right)$. In-

$$
(-t) \bar{\sigma}_{\Gamma}=\bar{\sigma}_{\Gamma}=\bar{\sigma}_{\Gamma}=\sum_{(a)}
$$

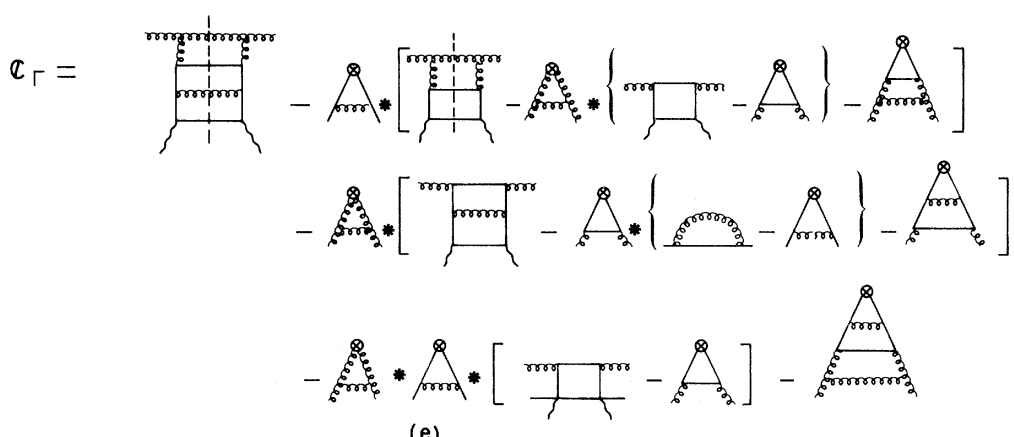

(e)

FIG. 12. Singlet DI graph of class $\left(\mathrm{a}_{2}\right)$ : (a) mass-factorization parts, (b) $R$ operation, (c) modified $R$ operation, (d) overall subtraction, (e) Wilson coefficient. 

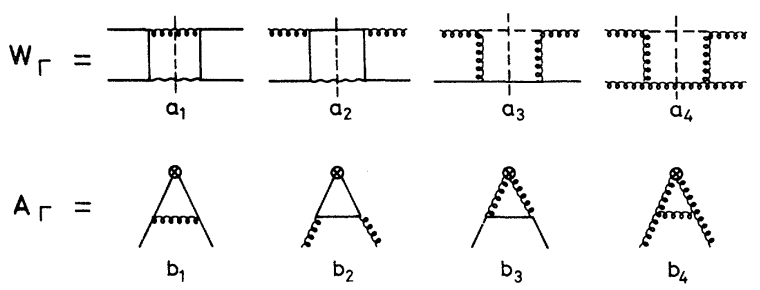

FIG. 13. Singlet DY cross sections $a_{1}-a_{4}$, and the corresponding OME's $b_{1}-b_{4}$.

stead of Eq. (4.10) we therefore can write [Fig. 10(e)]

$$
\bar{\sigma}_{\Gamma}^{\gamma q}=\sigma_{\Gamma}^{\gamma q}-\sigma_{\gamma_{1}}^{\gamma g} A_{\Gamma / \gamma_{1}}^{q g}
$$

The overall counterterm is, up to a finite renormalization, the same as in Eq. (4.11) leaving [see Fig. 10(f)]

$$
C_{\Gamma}^{q}=\sigma_{\Gamma}^{\gamma q}-A_{\Gamma / \gamma_{1}}^{q g}\left(\sigma_{\gamma_{1}}^{\gamma g}-A_{\gamma_{1}}^{g q}\right)-A_{\Gamma}^{q q}
$$

This expression corresponds precisely to what is found from the mass-factorization Eq. (4.3).

In Fig. 11 we give the result of an analogous analysis for a different cut graph. In order to demonstrate that our reasoning indeed extends to

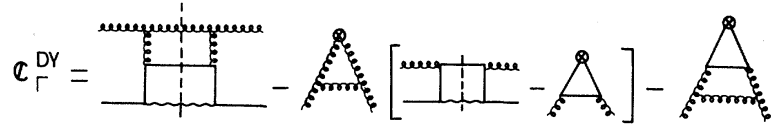

FIG. 15. Single DY graph of class $\left(a_{2}\right)$ (one mass singularity).

graphs of higher orders, we evaluate the cut graph shown in Fig. 12. The two mass-factorization parts $\gamma_{1}$ and $\gamma_{2}$ are indicated [Fig. 12(a)]. The (usual) $R$ operation with the scalar source reads [Fig. 12(b)]

$$
\bar{\sigma}_{\Gamma}^{\gamma g}=\sigma_{\Gamma}^{\gamma g}+\sigma_{\Gamma / \gamma_{1}}^{\gamma g}\left(-t^{\gamma_{1}} \bar{\sigma}_{\gamma_{1}}^{\gamma q}\right)+\sigma_{\Gamma / \gamma_{2}}^{s g}\left(-t^{\gamma_{2}} \bar{\sigma}_{\gamma_{2}}^{\gamma g}\right),
$$

whereas the modified $R$ operation reveals [Fig. 12(c)]

$$
\begin{aligned}
\bar{\sigma}_{\Gamma}^{\gamma g} & =\sigma_{\Gamma}^{\gamma g}+\sigma_{\Gamma / \gamma_{1}}^{\gamma g}\left(-t^{\gamma_{1}} \bar{\sigma}_{\gamma_{1}}^{\gamma q}\right)+\bar{\sigma}_{\gamma_{2}}^{\gamma g}\left(-t^{\Gamma / \gamma_{2}} \sigma_{\Gamma / \gamma_{2}}^{s g}\right) \\
& =\sigma_{\Gamma}^{\gamma g}-\sigma_{\Gamma / \gamma_{1}}^{\gamma g} A_{\gamma_{1}}^{q q}-\left(\sigma_{\gamma_{2}}^{\gamma g}-\sigma_{\gamma_{1}}^{\gamma q} A_{\gamma_{2} / \gamma_{1}}^{g q}\right) A_{\Gamma / \gamma_{2}}^{g g} .
\end{aligned}
$$

The overall counterterm is readily evaluated with the help of Fig. 12(d):

$$
\left(-t^{\Gamma}\right) \bar{\sigma}_{\Gamma}^{\gamma g}=-A_{\Gamma}^{g q}+A_{\Gamma / \gamma_{1}}^{g q} A_{\gamma_{1}}^{q q}+A_{\Gamma / \gamma_{2}}^{g g}\left(A_{\gamma_{2}}^{g q}-A_{\gamma_{2} / \gamma_{1}}^{g q} A_{\gamma_{1}}^{q q}\right)
$$

and the Wilson coefficient determined as [Fig. 12(e)]

$$
\begin{aligned}
\underset{\Gamma}{\mathbb{g}}= & \sigma_{\Gamma}^{\gamma g}-\left[\sigma_{\Gamma / \gamma_{1}}^{\gamma g}-A_{\Gamma / \gamma_{2}}^{g g}\left(\sigma_{\gamma_{2} / \gamma_{1}}^{\gamma g}-A_{\gamma_{2} / \gamma_{1}}^{g q}\right)-A_{\Gamma / \gamma_{1}}^{g q}\right] A_{\gamma_{1}}^{q q} \\
& -\left[\sigma_{\gamma_{2}}^{\gamma g}-A_{\gamma_{2} / \gamma_{1}}^{g q}\left(\sigma_{\gamma_{1}}^{\gamma q}-A_{\gamma_{1}}^{q q}\right)-A_{\gamma_{2}}^{g q}\right] A_{\Gamma / \gamma_{2}}^{g g}-\left(\sigma_{\gamma_{2} / \gamma_{1}}^{\gamma g}-A_{\gamma_{2} / \gamma_{1}}^{g q}\right) A_{\gamma_{1}}^{q q} A_{\Gamma / \gamma_{2}}^{g g}-A_{\Gamma}^{g g} .
\end{aligned}
$$

\section{DY examples (singlets)}

In Fig. 13 we give a set of primitively divergent DY cut graphs with the notation of DI scattering taken over: $W^{q q}\left(\mathrm{a}_{1}\right), W^{g q}\left(\mathrm{a}_{2}\right), W^{q g}\left(\mathrm{a}_{3}\right), W^{g g}\left(\mathrm{a}_{4}\right)$. The corresponding OME's are the same as in DI scattering [see Figs. 13( $\left.b_{1}\right)-13\left(b_{4}\right)$ and subsection IV B].

All primitively divergent graphs [class $\left(a_{1}\right)$, Fig. 13] are mass factorized as in DI scattering by sub-
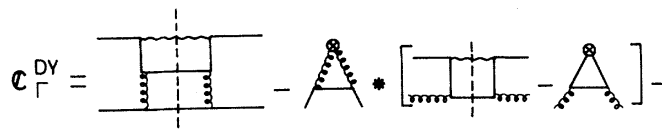

FIG. 14. Singlet DY graph of class $\left(a_{2}\right)$ (one mass singularity). tracting the OME from the cut graph [Eq. (4.9)]. In Figs. 14-16 we expose the results of a few more complicated examples of class $\left(a_{2}\right)$. The DY coefficient for the graph $\Gamma$ (successively chosen to be Figs. 14, 15, and 16) reads

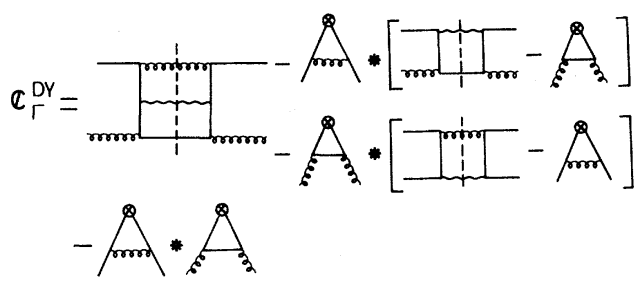

FIG. 16. Singlet DY graph (two mass singularities) of class $\left(a_{2}\right)$. 


$$
\begin{aligned}
& { }_{\Gamma}^{q q}=W_{\Gamma}^{q q}-A_{\Gamma / \gamma_{1}}^{q g}\left(W_{\gamma_{1}}^{g q}-A_{\gamma_{1}}^{g q}\right)-A_{\Gamma}^{q q}, \\
& { }_{\Gamma}^{\mathrm{\sigma} g q}=W_{\Gamma}^{g q}-A_{\Gamma / \gamma_{1}}^{g g}\left(W_{\gamma_{1}}^{g q}-A_{\gamma_{1}}^{g q}\right)-A_{\Gamma}^{g q}, \\
& { }_{\Gamma}^{g q}=W_{\Gamma}^{g q}-A_{\Gamma_{1}}^{q q}\left(W_{\Gamma_{2}}^{g q}-A_{\Gamma_{2}}^{g q}\right) \\
& -A_{\Gamma_{2}}^{g q}\left(W_{\Gamma_{1}}^{q q}-A_{\Gamma_{1}}^{q q}\right)-A_{\Gamma_{2}}^{g q} A_{\Gamma_{1}}^{q q} .
\end{aligned}
$$

\section{DIAGRAMMATIC MASS FACTORIZATION IN GAUGE THEORIES}

In this section, we increase the level of complexity and discuss two further complications, the infrared (IR) singularities and the gauge dependence, which can no longer be ignored if one aims for mass factorization in the gauge-theory framework. The Kinoshita-Lee-Nauenberg (KLN) theorem ${ }^{8}$ guarantees cancellation of all mass and IR singularities provided summation over all those initial and final states is carried out which are degenerate in energy. If the initial-state summation is dropped, unitarity enforces cancellation of all $M / \mathrm{IR}$ singularities originating from the final state. The cancellation of all IR divergences in the total parton cross section is a necessary condition for the validity of mass factorization. In QED the initial-state IR singularities are well known to compensate one another due to the BlochNordsieck (BN) theorem. ${ }^{11}$ In QCD, however, it only applies if there is no, or one, colored particle in the initial state. ${ }^{12}$ The IR singularities originating from initial states with two colored particles do not cancel any longer in order $g^{4}$, such as, for instance, in the DY process. ${ }^{13}$ Fortunately, as is shown by the authors of Ref. 13, the IR-singular terms are multiplied with factors which vanish in the Bjorken limit (higher-twist effects). Not much is known so far about the deeper reasons for this breakdown of the BN theorem. In particular, it is unknown whether in the higher-order contributions the IR singularities persist-even for lowest twist. All external IR singularities of the DI cut graphs are also found in their corresponding operator ma-
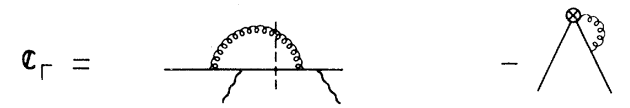

FIG. 17. Class $\left(a_{1}\right)$ graph (nonsinglet) in covariant gauge.

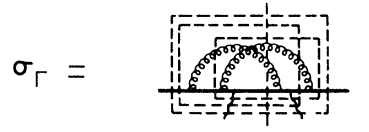

(a)

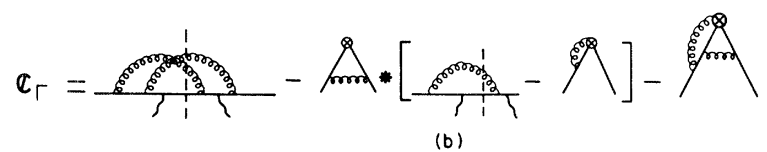

FIG. 18. Class $\left(a_{2}\right)$ graph (nonsinglet) in covariant gauge: (a) mass-factorization parts (except $\gamma_{2}$ ), (b) Wilson coefficient.

trix elements, and therefore their cancellation is expected in the Wilson coefficient. If the DY cross section reveals extra IR singularities they cannot be regularized by those of the operator matrix elements and the universality of mass factorization is broken.

We would like to attract the reader's attention to the importance of a correct mass assignment. The "conventional" off-shell mass choice, as shown in Ref. 14, leads to an inconsistent regularization of the $M /$ IR singularities which manifests itself most clearly when timelike and spacelike results are combined. A seeming breakdown of mass factorization in higher orders is the consequence; similar$1 y$, the lowest-order correction terms become dependent on the particularly chosen mass assignment and appear as arbitrary.

While working on QCD field theory, a second complication arises from the gauge freedom which influences the individual cut graphs. BPHZ mass factorization, developed and described for nongauge theories, applies in an analogous manner to QCD provided the axial gauge is chosen. ${ }^{15}$ This gauge is particularly suitable since the rules for assessing and classifying the mass (collinear) singularities can be taken over from the nongauge theories (see Sec. III). Choosing a different gauge (e.g., Feynman gauge) leads to a change in the $M$ singularity structure of each graph; some of them, $M$ finite in the axial gauge, become $M$ singular in a covariant gauge. BPHZ mass factorization, however, is still applicable. The "new" mass singularities appearing in a specific cut graph are subtracted by those of the corresponding OME which, of course, must be worked out in the same gauge.

Note that the number of operator graphs increases

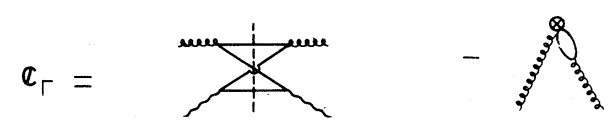

FIG. 19. Class $\left(a_{1}\right)$ graph (singlet) in covariant gauge. 


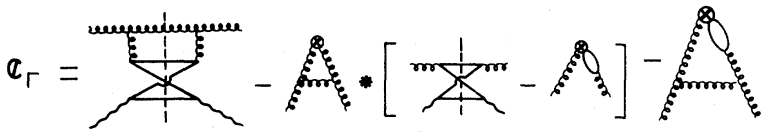

FIG. 20. Class $\left(a_{2}\right)$ graph (singlet) in covariant gauge.

if a gauge, different from the axial gauge, is chosen. For nonsinglet examples, we refer to Figs. 17 and 18, and similarly Figs. 19 and 20 illustrate singlet examples.

We caution the reader on the possible traps if, in a nonaxial gauge, the mass-factorization parts are determined. In Fig. 18(a), for example, $\gamma_{1}$ is a mass-factorization part, but not $\gamma_{2}$. Although both graphs have one gluon line attached to the external quark line, $\gamma_{2}$ is $\boldsymbol{M}$ finite. The reason is traced back to the $\gamma_{1}$ gluon line falling between the electromagnetic vertex and the strong vertex of $\gamma_{2}$. A similar situation (in renormalization) is found in the two-loop operator graph of Fig. 18(b). The triangular-vertex subgraph is the only renormalization part, the other subgraphs being $M$ finite. The reason again is found in the gluon line of the triangular subgraph which separates the operator vertex (indicated by $\otimes$ ) from the strong vertex.

\section{DY CORRECTION TERM WITH THE BPHZ METHOD}

In this section we determine the DY correction term $\Delta\left(1, \bar{g}^{2}\right)$ [defined in Eq. (2.6)] by a formalism analogous to BPHZ mass factorization of the DY coefficient function $C^{\text {DY }}$. Rewriting Eq. (2.6) in the (nonsinglet or singlet) form

$$
\Delta_{i j}\left(1, \bar{g}^{2}\right)=\left[\sigma_{i}\left(Q, p_{1}, \mu, g(\mu)\right) \sigma_{j}\left(Q, p_{2}, \mu, g(\mu)\right)\right]^{-1} W_{i j}\left(Q, p_{1}, p_{2}, \mu, g(\mu)\right),
$$

where $i, j$ stand for the quark-gluon indices, we observe a close analogy to DY mass factorization expressed by Eq. (4.8). In particular, $\mathfrak{E}_{i j} \mathrm{DY} \leftrightarrow \Delta_{i j}$ and $\left[\mathfrak{R}\left(p_{1}\right) \mathscr{U}\left(p_{2}\right)\right]^{-1} \leftrightarrow\left[\sigma_{i}\left(p_{1}\right) \sigma\left(p_{2}\right)\right]^{-1}$. ङ $\mathrm{DY}$ was obtained by factorizing the product of two OME's out of the DY cross section which in the sense of BPHZ takes the role of the mass counterterms. In the case of $\Delta_{i j}$, the role of the OME's is taken over by the DI cross sections $\sigma_{i}$ (conventionally chosen to be the parton structure function $\mathscr{F}_{2}$ ) now serving as counterterms for the DY cross section. As a result, the BPHZ technique with its diagrammat- ic presentation can, in complete analogy, be applied on Eq. (6.1); all OME's are simply replaced by the corresponding DI cut graphs.

Evaluation of the nonsinglet graphs is straightforward. The $R$ operation again defines the reduced cross section $\bar{W}_{\Gamma}$ recursively

$$
\bar{W}_{\Gamma}=W_{\Gamma}+\sum_{F} W_{F} \prod_{\tau=1}^{c}\left(-t^{\gamma_{c}}\right) \bar{W}_{\gamma_{c}}
$$

with the counterterms and the Taylor expansions related as

(a)

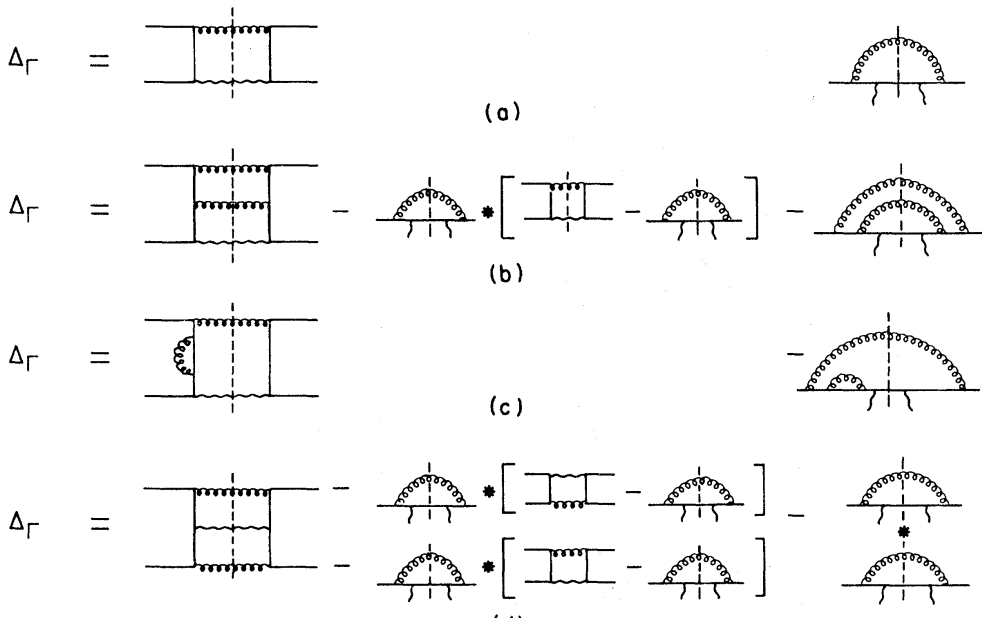

(d)

FIG. 21. DY correction term (nonsinglet): (a) class $\left(a_{1}\right)$ graph, (b) class $\left(a_{2}\right)$ graph, (c) class $\left(a_{3}\right)$ graph, (d) class $\left(a_{2}\right)$ graph (with two mass singularities). 


$$
\begin{aligned}
& \left(-t^{\Gamma}\right) W_{\Gamma}=-\left.\sigma_{\Gamma_{1}} \sigma_{\Gamma_{2}}\right|_{\Gamma_{1}, \Gamma_{2} \subset \Gamma}, \\
& \left(-t^{\Gamma}\right) \bar{W}_{\Gamma}=\left.\left[\sigma_{\Gamma_{1}} \sigma_{\Gamma_{2}}\right]^{-1}\right|_{\Gamma_{1}, \Gamma_{2} \subset \Gamma},
\end{aligned}
$$

$\Gamma_{1}$ and $\Gamma_{2}$ are the maximal mass-factorization parts of $\Gamma$. The DY correction term following from graph $\Gamma$ is given by

$$
\Delta_{\Gamma}=\left(1-t^{\Gamma}\right) \bar{W}_{\Gamma} \text {. }
$$

In Fig. 21 we illustrate our reasoning by several examples with

$$
\begin{aligned}
\Delta_{\Gamma=a_{1}}= & W_{\Gamma}-\sigma_{\Gamma}, \\
\Delta_{\Gamma=a_{2}}= & W_{\Gamma}-\sigma_{\gamma_{1}}\left(W_{\gamma_{1}}-\sigma_{\gamma_{1}}\right)-\sigma_{\Gamma}, \\
\Delta_{\Gamma=a_{3}}= & W_{\Gamma}-\sigma_{\Gamma}, \\
\Delta_{\Gamma=a_{4}}= & W_{\Gamma}-\sigma_{\Gamma_{1}}\left(W_{\Gamma_{2}}-\sigma_{\Gamma_{2}}\right) \\
& \quad-\sigma_{\Gamma_{2}}\left(W_{\Gamma_{1}}-\sigma_{\Gamma_{1}}\right)-\sigma_{\Gamma_{1}} \sigma_{\Gamma_{2}} .
\end{aligned}
$$

The letters $\left(a_{1}-a_{4}\right)$ point to the particular graph in Fig. 21. These results immediately follow from the examples illustrating DY mass factorization (Figs. 13-16) if the replacement OME $\rightarrow$ DI cross section is carried out. The procedure so far exposed is incomplete; it leads in general to an incorrect DY correction term. Inspection of its $g^{2}$ expansion reveals in order $g^{2} \Delta^{\mathrm{DY}}=\Delta_{1}$ and in order $g^{4} \Delta^{\mathrm{DY}}=\Delta_{2}+b_{1} \Delta_{1} \ln \left(-q^{2} / \mu^{2}\right)$. It appears that $\Delta_{2}$ receives contributions from graphs which belong to the classes $\left(a_{1}\right),\left(a_{2}\right)$, and $\left(a_{3}\right)$ (see Sec. III), whereas the second term $b_{1} \Delta_{1} \ln \left(-q^{2} / \mu^{2}\right)$ is exclusively built up from graphs out of class $\left(a_{3}\right)$. This last term is traced back to coupling-constant renormalization; it results from $g^{2}$ expansion of the running coupling constant $\bar{g}\left(Q^{2}\right)$. Pushing this reasoning to higher orders will introduce more and more of the $\beta$ coefficients of Eq. (2.8). It thus becomes clear why $\left(\mathrm{a}_{3}\right)$ graphs can only build up such terms; they contribute self-energy and strong-vertex corrections which renormalize the coupling constant. In order to proceed correctly we either introduce extra subtractions which remove the contribution $b_{1} \Delta_{1} \ln \left(Q^{2} / \mu^{2}\right)$ leading, however, to more complicated diagrammatic rules. As an alternative (which we favor), one simply sets $Q^{2}=\mu^{2}$. In this way, all unwanted terms disappear.

To deal with the singlet graphs, one follows the analogous procedure as exposed for DY mass factorization in subsections IV B and IV C. Again, we introduce the scalar external source and make use of the modified $R$ operation [see Eq. (4.6)]. However, in this case, the cut graphs describing the scalar-source-gluon interaction cannot be removed. In Fig. 22, we introduce a few singlet examples. The algebraic expression for Fig. 22(a) is derived from Eq. (4.20) with the OME replaced by the corresponding DI cross section. One finds

$$
\Delta_{\Gamma}^{q q}=W_{\Gamma}^{q q}-\sigma_{\Gamma / \gamma_{1}}^{s q}\left(W_{\gamma_{1}}^{g q}-\sigma_{\gamma_{1}}^{\gamma g}\right)-\sigma_{\Gamma}^{\gamma q}
$$

For a numerically explicit example we refer to Refs. 10 and 16. The algebraic expressions for Figs. 22(b) and 22(c) are derived from Eqs. (4.21) and (4.22). The diagrammatic form of $\Delta^{D Y}$ for the class $\left(\mathrm{a}_{3}\right)$ graphs follows from the nonsinglet DY graphs.

The deeper justification of the above rules goes back to DY mass factorization exposed by Eqs. (2.5) and (4.8). We replace everywhere the renormalization point by the off-shell photon mass

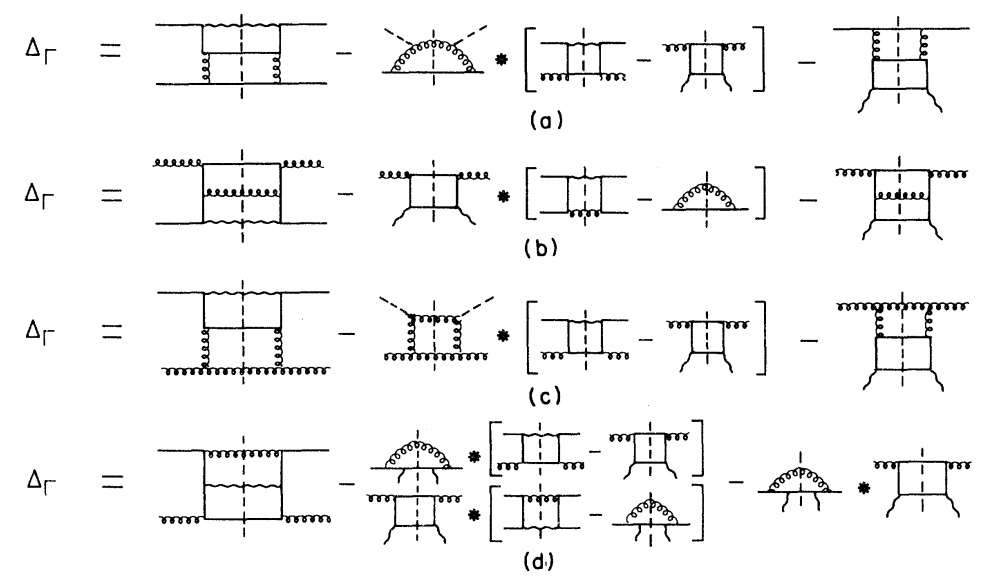

FIG. 22. DY correction term of class $\left(a_{2}\right)$ graphs (singlet). 
$\mu^{2}=Q^{2}$. Further, we assume that, due to the subtraction freedom in operator renormalization, the operator matrix elements $A\left(p_{i}{ }^{2}, g(\mu)\right)$ are specifically chosen so that for $\mu^{2}=Q^{2}$ the equality

$$
A\left(p_{i}^{2}, Q^{2}, g\left(Q^{2}\right)\right)=\sigma\left(p_{i}{ }^{2}, Q^{2}, g\left(Q^{2}\right)\right)
$$

holds. Going back to Eqs. (2.5) and (4.8) one therefore finds

$$
\begin{aligned}
\Delta\left(1, g\left(Q^{2}\right)\right) & =\left[\sigma\left(p_{1}^{2}, Q^{2}, g\left(Q^{2}\right)\right) \sigma\left(p_{2}{ }^{2}, Q^{2}, g\left(Q^{2}\right)\right)\right]^{-1} W\left(Q^{2}, p_{1}{ }^{2}, p_{2}{ }^{2}, Q^{2}, g\left(Q^{2}\right)\right) \\
& =1+g^{2}\left(Q^{2}\right) \Delta_{1}+g^{4}\left(Q^{2}\right) \Delta_{2}+\cdots,
\end{aligned}
$$

which justifies application of our earlier rules, in particular the introduction of the scalar external source in order to determine the coefficients $\Delta_{i}$.

\section{ALL-ORDER PROOF OF BPHZ MASS FACTORIZATION}

In the previous sections, we introduced the definitions and notions needed for the graph-bygraph BPHZ mass factorization, and we illustrated its reasoning by several examples. Having thus gained sufficient experience, we develop in this section an all-order proof of mass factorization. In order to facilitate a clear presentation of the important steps, we limit ourselves to the nonsinglet case. With slight modifications, the arguments can be carried over to the singlet graphs where, however, the notation becomes more complex.

In DI scattering we make use of the subgraph ordering: $\gamma_{1} \subset \gamma_{2} \subset \cdots \subset \gamma_{n} \equiv \Gamma$ where the massfactorization parts $\gamma_{i}=O\left(\left(g^{2}\right)^{i}\right)$. From Eqs. (3.7) and (3.10) we derive

$$
\begin{aligned}
\sigma_{\Gamma}^{(n)}= & \boldsymbol{\sigma}_{\Gamma}^{(n)}-\sum_{\gamma_{i}} \sigma_{\Gamma / \gamma_{i}}^{(n-i)}\left(-t^{\gamma_{i}}\right) \bar{\sigma}_{\gamma_{i}}^{(i)} \\
& -\left(-t^{\Gamma}\right) \bar{\sigma}_{\Gamma}^{(n)},
\end{aligned}
$$

where $\Gamma$ indicates a particular DI cut graph of order $\left(g^{2}\right)^{n}$, the order being indicated by the superscript $(n)$. The first term on the right-hand side is mass finite, the second term contains all ('t HooftVeltman $\left.{ }^{7}\right)$ overlapping divergences $\ln ^{i}\left(q^{2} / p^{2}\right)$ $\ln ^{j}\left(\mu^{2} / p^{2}\right)$, and the last one behaves as $\ln ^{i}\left(\mu^{2} / p^{2}\right)$.
We go back to Eq. (2.4) where the mass singularities of the DI cross section are expected to be canceled by the mass-singular function $A^{-1}$.

The cross-section form and the ansatz for $A^{-1}$ are

$$
\begin{aligned}
& \sigma=\sum_{n=0}^{\infty}\left(g^{2}\right)^{n} \sum_{\Gamma} \sigma_{\Gamma}^{(n)}, \\
& A^{-1}=\sum_{m=0}^{\infty}\left(g^{2}\right)^{m} \sum_{\Gamma}\left\{A_{\Gamma}^{(m)}\right\}^{-1} .
\end{aligned}
$$

The sum extends over all cut graphs $\Gamma$ contributing in a given order $n$ including the mass-finite contributions which are identified with the empty set $\varnothing\left[\sigma_{\phi}^{(n)}=\right.$ mass-finite contributions in order $\left.n\right]$. If mass factorization holds the product $A^{-1} \sigma$ [see Eq. (2.4)] must be free of any mass singularities and this in each order $n$; this has to be proven. The $\left(g^{2}\right)^{n}$ term of $A^{-1} \sigma$ [Eq. (2.4)] has the form

$$
\sum_{\Gamma}\left[\sigma_{\Gamma}^{(n)}+\left.\sum_{i=1}^{n}\left\{A_{\gamma_{i}}^{(i)}\right\}^{-1} \sigma_{\gamma_{n-i}}^{(n-i)}\right|_{\gamma_{i}, \gamma_{n-i}} \subset \Gamma\right] \text {, }
$$

where for the nonsinglet graphs $\Gamma / \gamma_{i} \equiv \gamma_{n-i}$. In Eq. (7.3) we have selected a graph $\Gamma$ of order $\left(g^{2}\right)^{n}$. All lower-order graphs can subsequently be generated via the mass-factorization parts $\gamma_{i}$ and formation of the reduced diagrams. In the product $A^{-1} \sigma$ we have regrouped terms such that each cut graph $\Gamma$ (of order $n$ ) is accompanied by the total sum of products: $\sigma_{\gamma_{n-i}}^{(n-i)}\left\{\boldsymbol{A}_{\gamma_{i}}^{(i)}\right\}^{-1}$ where all massfactorization parts satisfy $\gamma_{i}, \gamma_{n-i} \subset \Gamma$. Substituting $\sigma_{\Gamma}^{(n)}$ [given in Eq. (7.1)] into Eq. (7.3) leaves

$$
\sum_{\Gamma}\left[\mathfrak{\Xi}_{\Gamma}^{(n)}-\sum_{\gamma_{i}} \sigma_{\Gamma / \gamma_{i}}^{(n-i)}\left(-t^{\gamma_{i}}\right) \bar{\sigma}_{\gamma_{i}}^{(i)}-\left(-t^{\Gamma}\right) \bar{\sigma}_{\Gamma}^{(n)}+\left.\sum_{i=1}^{n}\left\{A_{\gamma_{i}}^{(i)}\right\}^{-1} \sigma_{\gamma_{n-i}}^{(n-i)}\right|_{\left.\gamma_{i} \gamma_{n-i} \subset \Gamma\right]}\right] .
$$

Using the identification

$$
\left.\sigma_{\Gamma / \gamma_{i}}^{(n-i)} \equiv \sigma_{\gamma_{n-i}}^{(n-i)}\right|_{\gamma_{n-i} \subset \Gamma}
$$

and the definition

$$
\left(-t^{\gamma_{i}}\right) \bar{\sigma}_{\gamma_{i}}=\left\{\left.\boldsymbol{A}_{\gamma_{i}}^{(i)}\right|_{\gamma_{i} \subset \Gamma}\right\}^{-1}
$$


all terms in Eq. (7.4) disappear apart from $\mathfrak{}_{\Gamma}$ which, as stated earlier near Eq. (3.9), is free of any mass singularities.

The proof of mass factorization for the DY process proceeds as in DI scattering. The product $\left[A_{1} A_{2}\right]^{-1} W$ must be shown to be mass finite in each order of $g$. The product of the two OME's has the form

$$
\left[A\left(p_{1}\right) A\left(p_{2}\right)\right]^{-1}=\sum_{m=0}^{\infty}\left(g^{2}\right)^{m} \sum_{k=0}^{m} \sum_{\gamma, \lambda}\left\{A_{\gamma}^{(k)}\left(p_{1}\right) A_{\lambda}^{(m-k)}\left(p_{2}\right)\right\}^{-1}
$$

and the DY cross section is similar to the DI cross section given by Eq. (7.2). We carry out the same regrouping as in DI scattering with the $\left(g^{2}\right)^{n}$ term of $\left[A_{1} A_{2}\right]^{-1} W$ given by

$$
\sum_{\Gamma}\left[W_{\Gamma}^{(n)}+\sum_{m=1}^{n} \sum_{k=0}^{m}\left\{A_{\gamma_{k}}^{(k)}\left(p_{1}\right) A_{\lambda_{m-k}^{(m-k)}\left(p_{2}\right)}^{(-1} W_{\Gamma /\left\{\gamma_{k}, \lambda_{m-k}\right.}\right\}\right]
$$

and the mass-factorization parts constrained by $\gamma_{k} \subset \Gamma_{1}, \lambda_{m-k} \subset \Gamma_{2} . \Gamma_{1}\left(\Gamma_{2}\right)$ is the maximal part of the DY cut graph $\Gamma$ containing the mass singularity $p_{1}{ }^{2}\left(p_{2}{ }^{2}\right) . \gamma_{k}, \lambda_{k}$ follow both the ordering as indicated near Eq. (7.1). Substituting the DY analog of Eq. (7.1) into (7.8), we obtain

$$
\begin{aligned}
& \sum_{\Gamma}\left[\mathfrak{\complement}_{\Gamma}^{(n)}-\sum_{v_{m}} W_{\Gamma / v_{m}}^{(n-m)}\left(-t^{v_{m}}\right) \bar{\sigma}_{v_{m}}^{(m)}-\left(-t^{\Gamma}\right) \bar{W}_{\Gamma}^{(n)}\right. \\
& +\sum_{m=1}^{n} \sum_{k=0}^{m}\left\{A_{\gamma_{k}}^{(n)}\left(p_{1}\right) A_{\left.\lambda_{m-k}^{(m-n)}\left(p_{2}\right)\right\}^{-1} W_{\Gamma /\left\{\gamma_{k}, \lambda_{m-k}\right\}} \mid \gamma_{k} \subset \Gamma_{1}, \lambda_{m-k} \subset \Gamma_{2}}\right] .
\end{aligned}
$$

We identify

$$
\left.W_{\Gamma / v_{m}}^{(n-m)} \equiv W_{\Gamma /\left\{\gamma_{k}, \lambda_{m-k}\right\}}\right|_{\gamma_{k} \subset \Gamma_{1}, \lambda_{m-k} \subset \Gamma_{2}}
$$

and use

$$
\left(-t^{v_{m}}\right) \bar{\sigma}_{v_{m}}^{(m)}=\left\{A_{\gamma_{k}}^{(k)}\left(p_{1}\right) A_{\lambda_{m-k}^{(m-k)}\left(p_{2}\right)}^{\left(m, \gamma_{k} \subset \Gamma_{1}, \gamma_{m-k} \subset \Gamma_{2}\right.}\right\}^{-1},
$$

where $\gamma_{k}, \lambda_{m-k}$ are the maximal parts of $v_{m}$, to find in Eq. (3.22) cancellation of all terms apart from ${ }^{C_{\Gamma}}$ which is mass finite.

We add some clarifying remarks. Cut graph $v_{m}$ (mass-factorization part of $\Gamma$ ) is of order $\left(g^{2}\right)^{m}$. In a chosen DY cut graph $\Gamma$ there are several subgraphs $v_{m} \subset \Gamma$ which are all of the same order. This is different from DI scattering where only one subgraph is of order $\left(g^{2}\right)^{m}$. To each DY subgraph $v_{m}$ corresponds one unique $\gamma_{k}$ and one unique $\lambda_{m-k}$ which automatically are its maximal parts. Let us, for example, consider a class $\left(a_{2}\right)$ graph of order $m$. In the DY process there are $(m+1)$ possible candidates, all of ladder type with $(m-k)$ gluon lines being exchanged above the virtual photon and $k$ below. In DI scattering, there is only one such possibility, i.e., the outer rainbow graph with $n$ rungs. The preceding arguments, however, apply only for nonsinglet graphs.

\section{SUMMARY AND CONCLUSIONS}

This paper develops an analogy between BPHZ renormalization and mass factorization. The UV singularities are replaced by the mass singularities and the role of the renormalization constant (associated with the Green's function) is taken over by the operator matrix element. Analysis of the mass singularities (similar to renormalization) reveals three essentially different classes: mass singular graphs giving rise to new counterterms, mass-finite graphs, mass-singular graphs with no new counterterms. We develop the mass analog of Zimmermann's forest technique. The massfactorization parts serve as graphical building blocks for Bogoliubov's $R$ operation in order to remove the overlapping divergences. Finally, we proceed to the Taylor expansion in order to determine the overall counterterm. The mass-factorized 
parton cross section (Wilson coefficient) then trivially follows.

Our reasoning is illustrated by several examples of nonsinglet DI/DY cut graphs and the massfactorized cross section is determined. Extension of this method to singlet graphs is, after the introduction of a (spurious) scalar external source, straightforward. Again, several illustrative DI/DY singlet examples are presented. The method, so far, was limited to nongauge theories. We therefore discuss in a separate section the subtleties which will arise in any gauge-theory framework. In particular, we discuss the IR singularities, their off-shell regularization and correct cancellation; and we expand on the changes if nonaxial gauges are used. The forest technique can equally be used to specify the DY correction term $\Delta_{i j}^{\mathrm{DY}}$; several examples explain the reasoning.

Having gained some experience of how to carry out, graph by graph, mass factorization, our study leads to a general, all-order proof of mass factorization. Apart from its (solid) proof by an alternative method, mass factorization can be presented graphically. Our method permits elegant determination of (i) all logarithmic coefficients of an order- $\left(g^{2}\right)^{n}$ cut graph once the lower-order graphs are fully known and the order- $\left(g^{2}\right)^{n}$ operator matrix elements are specified up to the logarithmic terms ${ }^{17}$; (ii) the DY coefficients function $\Delta(1, \bar{g})$ giving information on its $\ln ^{i}(1-\tau)$ expansion; and (iii) the determination of the DI Wilson coefficient and its DY analog in a transparent way. Point (i) is limited to the logarithmic singularities, in particular, the mass singularities which appear exclusively in the $2 P R$ graphs. Therefore, all 2 PI graphs have to be evaluated by a direct computation of the cut graphs. Points (i) and (ii) lead us to wonder as to the validity of our reasoning for the higher- twist terms and other fundamental features of mass factorization.
*Present address: Department of Physics, CERN, CH1211 Geneva 23, Switzerland.

1H. D. Politzer, Nucl. Phys. B129, 301 (1977).

2(a) C. T. Sachrajda, Phys. Lett. 73B, 185 (1978); (b) D. Amati, R. Petronzio, and G. Veneziano, Nucl. Phys. B140, 54 (1978); B146, 39 (1978); (c) R. K. Ellis, H. Georgi, M. Machacek, H. D. Politzer, and G. G. Ross, Phys. Lett. 78B, 281 (1978); Nucl. Phys. B152, 285 (1979); (d) S. Libby and G. Sterman, Phys. Lett. 78B, 618 (1978); (e) J. Antoniades, L. Baulieu, and K. Kounnas, Nucl. Phys. B168, 394 (1980).

${ }^{3}$ F. J. Dyson, Phys. Rev. $\underline{75}, 486$ (1949); 75, 1736 (1949); A. Salam, ibid. $\underline{82}, 217$ (1951); $\underline{84}, 436$ (1951); J. D. Bjorken and S. D. Drell, Relativistic Quantum Fields (McGraw-Hill, New York, 1965).

4(a) A. H. Mueller, Phys. Rev. D 9, 963 (1974); 18, 3705 (1978); A. H. Mueller and S. Gupta, ibid. 20, 118 (1979); (b) G. Curci, W. Furmanski, and R. Petronzio, Nucl. Phys. B175, 27 (1980).

${ }^{5}$ B. Humpert and W. L. van Neerven, Phys. Lett. 102B, 426 (1981); Report No. CERN-TH3086 (unpublished).

6 (a) N. N. Bogoliubov and D. V. Shirkov, Introduction to the Theory of Quantized Fields (Interscience, New York, 1959); (b) W. Zimmermann, Lectures on Elementary Particles and Quantum Theory, 1970 Brandeis University Summer Institute in Theoretical Physics (MIT Press, Cambridge, Mass., 1971), Vol. 1, p. 528; (c) K. Hepp, Commun. Math. Phys. 2, 301 (1966); (d) N. N. Bogoliubov and O. Parasiuk, Acta Math. 97, 227 (1957).

${ }^{7}$ G. 't Hooft and M. Veltman, Nucl. Phys. B44, 189 (1972); see also their paper in Particle Interactions at Very High Energies, edited by D. Speiser, F. Halzen, and J. Weyers (Plenum, New York, 1979), Part 8, p. 177.

${ }^{8}$ G. T. Bodwin, S. J. Brodsky, and G. P. Lepage, Phys. Rev. Lett. 47, 1799 (1981); A. H. Mueller, Columbia University Report No. CU-TP-213, 1981 (unpublished).

${ }^{9}$ T. Kinoshita, J. Math. Phys. $\underline{3}, 650$ (1962); T. D. Lee and M. Nauenberg, Phys. Rev. 133, B1549 (1964); N. Nakanishi, Prog. Theor. Phys. 19, 159 (1958).

${ }^{10}$ P. M. Fishbane, C. S. Lam, and T. M. Yan, Phys. Rev. D 20, 2960 (1979); A. N. Schellekens and W. L. van Neerven, ibid. 21, 2619 (1980).

${ }^{11}$ F. Bloch and A. Nordsieck, Phys. Rev. 52, 54 (1932); 52, 59 (1932); D. R. Yennie, S. C. Frautschi, and H. Suura, Ann. Phys. 13, 379 (1961).

${ }^{12}$ S. B. Libby and G. Sterman, Phys. Rev. D $\underline{19}, 2468$ (1979).

${ }^{13}$ R. Doria, J. Frenkel, and J. C. Taylor, Nucl. Phys. B168, 93 (1980); A. Andrasi, M. Day, R. Doria, J. Frenkel, and J. C. Taylor, ibid. B182, 104 (1981); C. Di'Lieto, S. Gendron, I. G. Halliday, and C. T. Sachrajda, ibid. B183, 223 (1981).

${ }^{14}$ B. Humpert and W. L. van Neerven, Phys. Lett. $\underline{89 B}$, 69 (1979); Nucl. Phys. B178, 498 (1981); Nucl. Phys. B184, 225 (1981).

${ }^{15}$ W. Kummer, Acta Phys. Austriaca 41, 315 (1975); W. Kainz, W. Kummer, and M. Schweda, Nucl. Phys. B79, 484 (1974); W. Konetschny and W. Kummer, ibid. B100, 106 (1975); B108, 397 (1976); B124, 145 (1977).

${ }^{16}$ A. P. Contogouris and J. Kripfganz, Phys. Rev. D 20, 2295 (1979).

${ }^{17}$ B. Humpert and W. L. van Neerven (unpublished). 